\title{
Adaptive Transmission Range Based Topology Control Scheme for Fast and Reliable Data Collection
}

\author{
Haojun Teng $\mathbb{D},{ }^{1}$ Kuan Zhang, ${ }^{2}$ Mianxiong Dong $\mathbb{D},{ }^{3}$ Kaoru Ota, ${ }^{3}$ Anfeng Liu $\mathbb{D},{ }^{1,4}$ \\ Ming Zhao, ${ }^{5}$ and Tian Wang ${ }^{6}$ \\ ${ }^{1}$ School of Information Science and Engineering, Central South University, Changsha 410083, China \\ ${ }^{2}$ Department of Electrical and Computer Engineering, University of Nebraska, Lincoln, USA \\ ${ }^{3}$ Department of Information and Electronic Engineering, Muroran Institute of Technology, Japan \\ ${ }^{4}$ The State Key Laboratory of Industrial Control Technology, Zhejiang University, Hangzhou 310027, China \\ ${ }^{5}$ School of Software, Central South University, Changsha 410083, China \\ ${ }^{6}$ School of Computer Science, National Huaqiao University, Quanzhou 362000, China \\ Correspondence should be addressed to Anfeng Liu; afengliu@mail.csu.edu.cn
}

Received 29 April 2018; Accepted 2 July 2018; Published 12 July 2018

Academic Editor: Sajid Hussain

Copyright (C) 2018 Haojun Teng et al. This is an open access article distributed under the Creative Commons Attribution License, which permits unrestricted use, distribution, and reproduction in any medium, provided the original work is properly cited.

\begin{abstract}
An Adaptive Transmission Range Based Topology Control (ATRTC) scheme is proposed to reduce delay and improve reliability for data collection in delay and loss sensitive wireless sensor network. The core idea of the ATRTC scheme is to extend the transmission range to speed up data collection and improve the reliability of data collection. The main innovations of our work are as follows: (1) an adaptive transmission range adjustment method is proposed to improve data collection reliability and reduce data collection delay. The expansion of the transmission range will allow the data packet to be received by more receivers, thus improving the reliability of data transmission. On the other hand, by extending the transmission range, data packets can be transmitted to the sink with fewer hops. Thereby the delay of data collection is reduced and the reliability of data transmission is improved. Extending the transmission range will consume more energy. Fortunately, we found the imbalanced energy consumption of the network. There is a large amount of energy remains when the network died. ATRTC scheme proposed in this paper can make full use of the residual energy to extend the transmission range of nodes. Because of the expansion of transmission range, nodes in the network form multiple paths for data collection to the sink node. Therefore, the volume of data received and sent by the near-sink nodes is reduced, the energy consumption of the near-sink nodes is reduced, and the network lifetime is increased as well. (2) According to the analysis in this paper, compared with the CTPR scheme, the ATRTC scheme reduces the maximum energy consumption by $9 \%$, increases the network lifetime by $10 \%$, increases the data collection reliability by $7.3 \%$, and reduces the network data collection time by $23 \%$.
\end{abstract}

\section{Introduction}

Topology control technology has been widely applied to wireless networks such as mobile ad hoc network [1-3], wireless sensor network, and emerging mobile networks $(E M N)$ to reduce the communication interference and energy consumption through altering the underlying network [48]. Topology control technology mainly adjusts the transmission power of communication devices to adjust their communication radius so as to achieve the goal of improving network performance. Topology control technology can be fully applied in wireless sensor networks based on the fact that the transmission power of a wireless sensor node is adjustable. The paper [9] pointed out that the sensor nodes have multiple power levels which can be adjusted. Topology control technology brings benefits to the network. (1) Reducing the energy consumption of nodes [10-14]: in wireless sensor networks, the density of communication devices is usually large [15]. When communication between devices to devices (D2D) is required, the transmission power can be adaptively adjusted according to the distance between devices (or nodes). Since the energy consumption of nodes is related to the communication distance to the second power (even to the fourth power), the adaptive adjustment of the 
transmission power of nodes according to the communication distance can effectively reduce the energy consumption of the nodes and extend the network lifetime. (2) Reducing network communication interference, thereby increasing the number of simultaneous network communications, improving network throughput, and at the same time reducing energy consumption [16-18]: if the nodes density is relatively high, appropriately reducing the communication radius of nodes will not only reduce the energy consumption, but also reduce the wireless communication interference between the nodes. Therefore, more nodes can communicate at the same time, thereby increasing the network's throughput.

Most of the topology control studies are based on the ideas mentioned above, that is, adaptively adjusting the communication radius of the nodes, and meanwhile satisfying the communication, the nodes communicate with the smallest possible communication radius. This can reduce energy consumption and interference. Increase throughput improves network life. These studies can be found in [10, 19-21]. Although the main idea of topology control has been widely used in most networks, it leads to good results. However, we have found that for a type of delay and loss sensitive data aggregation wireless sensor networks, the idea mentioned above cannot lead to good results. In some applications, wireless sensor networks need to periodically collect information such as crop temperature and humidity. The users of these applications need to know the minimum value or maximum value rather than every detail value. Therefore, using data aggregation can minimize the volume of data to transmit. In such data collection, any $\mathrm{n}$ data packets will be aggregated into one data packet. But the wireless channel is usually lossy due to the complexity of the physical environment. Less delay time of data collection is important for these applications. But more delay time will be caused by the packet loss if the wireless channel is lossy. Therefore, how to adopt the appropriate topology control technology in such applications to achieve fast, efficient, and highly reliable data collection is a challenging issue $[22,23]$.

Using broadcast method can effectively improve the reliability of data collection [24]. When the sender node transmits data by broadcasting, all the nodes which are in the transmission range of the sender node will receive the data possibly. Only when all the nodes fail to receive the data, the data will be lost. Obviously, this approach can significantly reduce the probability of packet loss but increases energy consumption. However, compared to retransmission method in unicast mode, broadcast still has the following advantages: (a) the sender node only needs to broadcast once and it may achieve high reliability. But the data loss in unicast mode requires multiple retransmissions. (b) Broadcast has a great advantage in data collection speed. In unicast mode, if the packet is lost, retransmission is required, and the retransmission time is very long. But broadcast mode usually does not need retransmission, so it reduces the transmission time.

There are a few studies based on the collection of broadcast data for delay and loss sensitive wireless sensor network. But according to our investigation, there is no topology control method based on broadcast data collection mode. The previous topology control method is to reduce the transmission radius of the nodes as much as possible to save energy and reduce the interference range. However, topology control in this article takes the exact opposite of the previous topology control strategy. In the topology control of this paper, instead of reducing the transmission radius of the nodes, the remaining energy in the network is fully utilized to increase the transmission radius of the nodes to improve the reliability of data collection and the data collection performance, while ensuring a high network lifetime. In summary, the main contributions of this article's research work are as follows:

(1) An Adaptive Transmission Range based Topology Control (ATRTC) scheme is proposed to reduce delay and improve reliability for data collection in delay and loss sensitive wireless sensor network. In contrast to the previous reduction of communication radius to reduce interference and save energy, the core idea of the ATRTC scheme is to increase the radius of broadcast to speed up the collection of data and improve the reliability of data collection. The reason for increasing the radius of broadcast to optimize the network performance is as follows: the expansion of the transmission range will allow the data packet to be received by more receivers, thus improving the reliability of data transmission. On the other hand, by extending the transmission range, data packets can be transmitted to the sink with fewer hops. Thereby the delay of data collection is reduced and the reliability of data transmission is improved. Extending the transmission range will consume more energy. Fortunately, we found the imbalanced energy consumption of the network. There is a large amount of energy remains when the network died. ATRTC scheme proposed in this paper can make full use of the residual energy to extend the transmission range of nodes. Because the expansion of transmission range, nodes in the network form multiple paths for data collection to the sink node. Therefore, the volume of data received and sent by the near-sink nodes is reduced, the energy consumption of the near-sink nodes is reduced, and the network lifetime is increased as well.

(2) According to the analysis in this paper, compared with the CTPR scheme, the ATRTC scheme reduces the maximum energy consumption by $9 \%$, increases the network lifetime by $10 \%$, increases the data collection reliability by $7.3 \%$, and reduces the network data collection time by $23 \%$.

The rest of the paper is organized as follows. Section 2 reviews related works comparing with our scheme. Section 3 describes the network model and defines problem statements of this paper. In Section 4, we give the design ATRTC scheme for delay and loss sensitive WSNs. In Section 5, we do performance analysis to ATRTC scheme. We conclude this paper in Section 6.

\section{Related Work}

Advances in technology have made sensors smaller, more accurate, and less expensive. Thanks to this, wireless sensor networks have been widely used in various fields [25-30]. Because building a sensor network requires a large number of sensor nodes, sensor nodes are usually cheap. Due to the cost, the battery, CPU, memory, and communication capabilities of 
sensor nodes are limited. The main goal of the optimization of the sensor network is to increase the lifetime and reliability of the sensor network and reduce the sensor delay [25, 31-33].

Topology control technology is an important and commonly used optimization technique in networks fields [3437]. Topology control technology has considerable studies in WSNs [38, 39]. At present, studies on topology control in WSNs can be mainly divided into network coverage issues and network connectivity issues. And network connectivity issues include Power Management Mechanisms and Power Control Mechanisms [9]. This paper focuses on the Power Control Mechanisms. In general, sensor nodes such as the MICA2 mote have multiple power levels adjustable. By such a fact, one natural question is the following: Is it wise to make radio work with the maximum working power all the time? Research literature later found that the answer is negative. The optimal transmission power is normally between minimum value and the maximum value. The paper [19] proposed a COMPOW Protocol. It can determine the minimum common transmitting range needed to ensure network connectivity. The authors believe that the two nodes that are closer together do not need the maximum transmission power. Reducing the transmission power to reduce the transmission range has two advantages. On the one hand, it can increase the battery lifetime. On the other hand, reducing the transmission radius can reduce the interference of neighbor nodes. It can increase the number of nodes that can transmit at the same time, thereby increasing the network capacity. But in reality, link is not always reliable, and it is difficult to guarantee the link quality under the transmission power of COMPOW. A large number of experimental results in [40] proved that the transmission power is related to the link quality. The relationship of transmission power and received signal strength indicator (RSSI)/link quality indicator (LQI) are analyzed, and a prediction model is established. The authors also proposed the Adaptive Transmission Power Control (ATPC) algorithm, a feedback-based transmission power control algorithm to dynamically maintain individual link quality over time. This algorithm achieves energy efficiency and guarantees the link quality between neighbors. However, the implementation of this algorithm also requires additional data exchanges between neighbor nodes. Therefore, although ATPC has improved in energy saving, the network delay will be increased.

For the issue of link quality, [41] conducted deep research. Marco and Bhaskar use mathematical techniques from communication theory to model and analyze low power wireless links. And they analyzed how multiple factors affect link quality. The paper [41] measured the link quality using packet reception rate $(\mathrm{PRR})$ and introduced the relationship between PRR and distance under certain transmission power conditions. Based on this, this paper analyzes the relationship between transmission distance and the power under the condition of guaranteeing link quality. In applications requiring high reliability, it is not wise to simply increase the link quality by increasing the transmission power. Because the relationship between transmit power and PRR is nonlinear, in the case of relatively high PRR, a huge transmission power improvement can only be obtained in exchange for a slight PRR improvement. In the application of high reliability requirements, the following methods are generally used to improve the reliability of the transmission:

(1) Broadcast technology: broadcast technology is a highly reliable data transmission technology. It allows nodes to transmit data to multiple destination nodes at the same time. As long as one destination node receives data, it can continue to transmit. Therefore, even if the quality of links between nodes is relatively low, the probability of successful transmission in the case of a large number of destination nodes can reach a very high level. Of course, broadcast technologies also have drawbacks. Uncontrolled broadcast technologies such as flooding can significantly increase the amount of data, cause a lot of energy waste, and shorten the network lifetime. This is called broadcast problems [42]. There are many papers on the research of broadcast storm problems and propose improvement strategies. The paper [43] introduces a location aided algorithm to compute the optimal local cover set without delay and without much communication overhead. The approach in [44] is based on selecting a small subset of nodes to form a forward node set to carry out a broadcast process. These methods in $[43,44]$ require each node to know its k-hop $(\mathrm{k}>=2)$ neighbor information, which cause a large amount of extra information exchange, which generates additional energy consumption, so the improvement of energy efficiency is not obvious. An important solution to the broadcast storm problem is to use data aggregation technology. The paper [45] shows that appropriate use of in-network aggregation can significantly reduce the amount of traffic generated over the network. Data aggregation technology is a very important technology to reduce the data volume in wireless sensor networks $[46,47]$. There are many researches on the application of data aggregation in wireless sensor networks. The paper [48] adopted the data aggregation technology in industrial wireless sensor networks (IWSNs). And it used cluster heads to reduce the amount of transmissions required to transmit the same information to the BS and reduces the latency at the expense of some reduction in performance. The paper [49] proposed distributing delay efficient data aggregation scheduling to reduce the delay effectively, in which data aggregation is an essential operation.

(2) Retransmission mechanism: in order to achieve high reliability, using the retransmission mechanism is the most direct choice. In the process of data transmission between the node and the destination node, if there is a packet loss, the destination node will send a message to the source node to inform the packets loss. Then, the source node will retransmit the lost packets. If the destination node retransmits successfully, it will return an ACK message. Otherwise, the source node will continue to retransmit and stop retransmitting until it succeeds or reaches the upper limit of the number of retransmissions. Many studies have achieved robust and reliable wireless transmission by using a retransmission mechanism. The papers $[50,51]$ are examples of using retransmission mechanisms to solve reliability problems. The paper [50] proposes PSFQ (Pump Slowly, Fetch Quickly), a reliable transport protocol suitable for a new class of reliable data applications in wireless sensor networks. 
The paper [51] proposes a scalable framework for reliable downstream data delivery that is specifically designed to both address and leverage the characteristics of a wireless sensor network, while achieving the reliability in an efficient manner.

The use of the retransmission mechanism is energy efficient compared to the broadcast mechanism and the following redundancy mechanism, but there are also problems with large delays, because the retransmission mechanism requires multiple transmissions and an acknowledgment message which lead to additional wait time.

(3) Redundancy scheme is also a way to improve reliability [52]. This scheme is usually using Erasure Coding, that is, when transmitting data, encode $M$ source packets into $N+R$ $(N+R>M)$ packets for transmission. At the destination node, it can reconstruct $M$ original messages if the destination node receives at least $N$ out of $N+R$ encoded data packets [53]. Theoretical analysis in [53] indicates that when the loss probability remains low or moderate, redundancy scheme is more reliable and energy efficient than retransmission. However, the performance of the redundancy scheme will greatly decline under the condition that the probability of loss is high.

\section{The System Model and Problem Statement}

3.1. The Network Model. A wireless sensor network for periodic data collection is adopted in this paper. This network is composed of a sink node and $n$ sensor nodes. The sensor node in the network is responsible for collecting the physical characteristics of the surrounding environment and generating data. On the other hand, the sensor node is also responsible for data transmission and data aggregation. The sink node in the network is responsible for collecting all incoming data and processing it for sending to users using the wireless sensor network. This kind of network has widely application scenarios, such as predicting the weather by collecting wind information periodically and monitoring forest fires by collection temperature information periodically. For the deployment of the network, the coverage of the entire network is a circle with radius $R$. The sink node is located in the center of the network, and $n$ sensor nodes are randomly and uniformly deployed in the entire network range with a distribution density of $\rho$.

3.2. The Wireless Channel Reliability Model. Due to the complexity of the physical environment, the communication links between nodes are lossy. This paper uses the packet receiving rate proposed by [41] to measure the quality of communication links between nodes. For a data transmission process, the sender is the node that sends the data, and receiver is the node that receives the data. The packet acceptance rate of a node is defined as the ratio of the number of data received by the receiver to the number of data sent by the sender node. According to [41], the packet reception rate (PRR) is calculated as follows:

$$
p=\left(1-\frac{1}{2} \exp ^{-(S N R / 2)\left(B_{N} / V_{s}\right)}\right)^{8 f}
$$

where $f$ is the frame size of the packet, $B_{N}$ is the noise bandwidth, and $V_{s}$ is the sending rate of nodes. In general,
TABLE 1: Parameters of sensor node.

\begin{tabular}{lcc}
\hline Symbol & Description & Value \\
\hline$V_{s}$ & Sending rate & $19.2 \mathrm{kbps}$ \\
$V_{r}$ & Receiving rate & $19.2 \mathrm{kbps}$ \\
$E_{\text {init }}$ & The initial energy of node & $2 \mathrm{~J}$ \\
$d_{0}$ & The reference distance & $5 \mathrm{~m}$ \\
$P_{n}$ & The noise floor & $-115 \mathrm{dBm}$ \\
$n$ & The path loss exponent & 4 \\
$f$ & The frame size of a data packet & $50 \mathrm{~B}$ \\
$B_{N}$ & The noise bandwidth & $30 \mathrm{HZ}$ \\
\hline
\end{tabular}

the values of $f, B_{N}$, and $V_{s}$ are fixed in the same network, and their specific values are summarized in Table 1. Substituting specific values of $f, B_{N}$, and $V_{s}$ into (1), the equation of $p$ with respect to $\gamma(d)$ can be expressed as

$$
p=\left(1-\frac{1}{2} \exp ^{-(\gamma(d) / 2)(1 / 0.64)}\right)^{8 f}
$$

Assuming a given transmit power $P_{t}$, the signal-to-noise ratio $\gamma$ at the distance $d$ can be expressed as

$$
\gamma(d)=P_{t}-P L(d)-P_{n}
$$

where Bottom noise $P_{n}$ is $-115 \mathrm{dBm}$ and $P L(d)$ is the loss value during transmission.

The value of $P L(d)$ can be derived from the following equation:

$$
P L(d)=P L\left(d_{0}\right)+10 n \log _{10}\left(\frac{d}{d_{0}}\right)+X_{\delta}
$$

where $d_{0}$ is a reference distance, $n$ is the path loss exponent, and $X_{\delta}$ is a zero-mean Gaussian distribution of the random variable.

3.3. The Data Collection Model. Wireless communication technology is adopted during the data transmission between nodes in the network. The routing algorithm used for the network is the shortest path algorithm. If the sink node is within the transmission range of the node, the data can be directly transmitted to the sink node. For a node that is far away from the sink node, the data need to be transmitted to a node that is closer to the sink node and forwarded by this node to a node closer to the sink node until it reaches the sink. This type of transmission is called multihop transmission. In the process of data transmission to the sink node, the number of transmissions is called the number of hops. For nodes with the same number of hops, their distribution positions appear as a ring, which we call a tier.

If all the nodes in the network have the same transmission range, the network can be divided into several tiers with the same width. If the ring width is $d(0<d \leq r)$, the network can be divided into $\lceil R / d\rceil$ tiers. From the center to number each tier in ascending order, $1,2,3, \cdots,\lceil R / d\rceil$, and $T_{i}$ denoted the nodes in tier $i$.

The data collection in the network refers to the process of collecting and processing the data packets generated by all the 


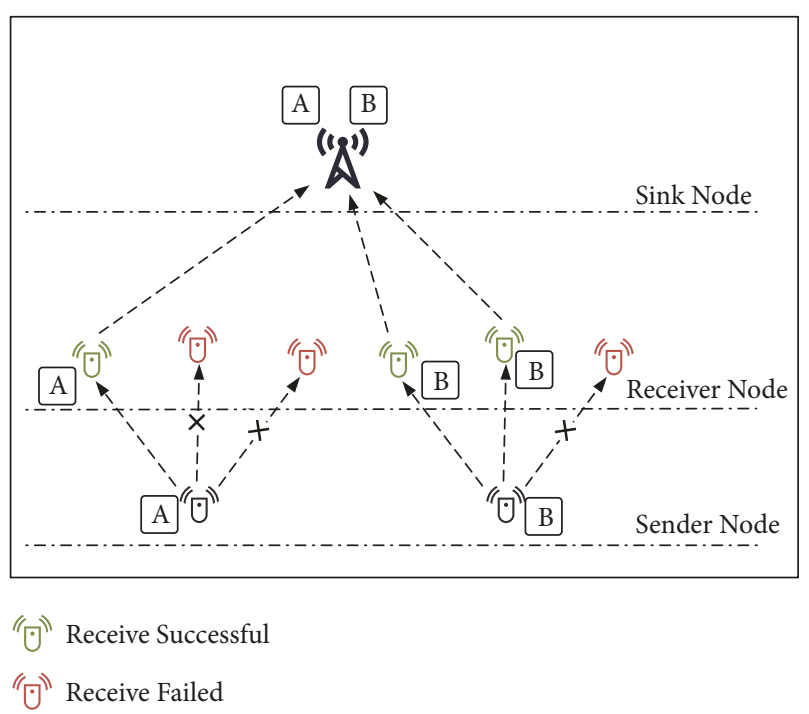

Figure 1: Broadcast mechanism.

sensor nodes in the network and collected in the sink node. The entire data collection process begins with the node in the outermost tier, from the outside to the inside, and the data are collected tier by tier according to a certain collection method until all the data reach the sink node.

The broadcast mechanism is adopted for data transmission in order to improve the reliability. By using the broadcast mechanism, sender node can send data to multiple receiver nodes simultaneously. As long as any node in the receiver nodes receives the data sent by the sender, it can ensure that the data continue to be transmitted without loss. As can be seen from Figure 1, there are two sender nodes sending data. But the data sent by them are not received by all receiver nodes. Although the data sent by the sender on the left is only received by one receiver node, the data can still be transmitted to the sink node. Therefore, using broadcasting can greatly improve the reliability of transmission. In general, the sink node has multiple antennas; therefore, its children can be seen as having multiple parent nodes.

Using broadcast mechanism improves the reliability of data transmission, but it dramatically increases the amount of data transmission as well. As can be seen from Figure 1, after a data packet $B$ sent by a sender node is received by two receiver nodes, both receiver nodes will send B to their parent node (the sink node); i.e., their parent node receives duplicate data causing energy waste. It is obviously not feasible to simply use the broadcast mechanism. Therefore, data aggregation technology is adopted in the process of network transmission. In the process of data collection, the node can use the data aggregation technology to process the received data, and the data size after processing will be greatly reduced.

Data aggregation technology is a technology used in data transmission to reduce the amount of data transmitted. As shown in Figure 2, the data aggregation technology uses a specific aggregate function to process the $m$ input data packets and outputs an aggregated data packet. The size of the output data packet is significantly reduced compared to

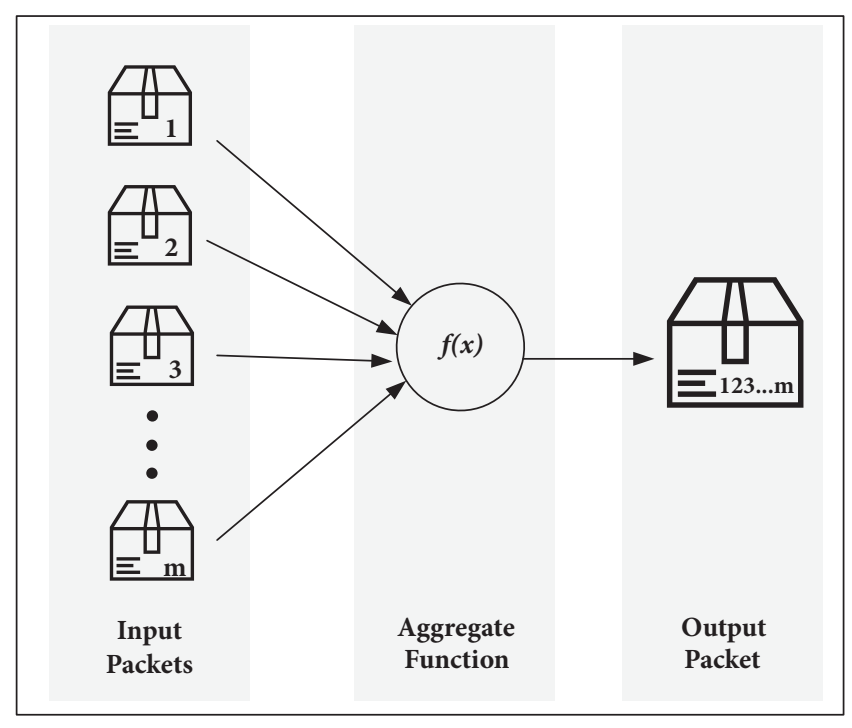

FIGURE 2: Data aggregation mechanism.

the total size of the input data packet. In this paper, a widely used aggregation model called increasing aggregation model is adopted, same as [54]. When $m$ raw packets are aggregated, the size of the aggregated packet can be calculated according to the following equation:

$$
\left|\vartheta^{\prime}\right|=\left|\vartheta_{1}\right|+\varepsilon \cdot\left(\left|\vartheta_{2}\right|+\left|\vartheta_{3}\right|+\cdots+\left|\vartheta_{m}\right|\right)
$$

where $\left|\vartheta^{\prime}\right|$ denotes the size of output packet after aggregation and $\left|\vartheta_{1}\right|,\left|\vartheta_{2}\right|, \cdots,\left|\vartheta_{m}\right|$ denote the size of $m$ raw input packets, respectively.

3.4. Problem Statement. For wireless sensor networks, how to achieve better network performance under limited resources is of great importance. For periodic data collection WSNs, improving the reliability of data collection, how to reduce the length of data collection, and how to improve the network life are the main aspects of our efforts to improve.

(1) Data collection time $(\mathscr{T})$ : data collection time is the time length for a data collection cycle. It is the time length from which the outermost node starts to transmit data to all nodes in the network to complete the data transmission. The longer the data collection time is, the longer it takes the user to get the latest data. Obviously, the shorter the data collection time, the better. Let $t_{i}$ denote the transmission time of all the nodes in tier $i$, and the data collection time $(\mathscr{T})$ can be expressed as the sum transmission time of nodes in all tiers:

$$
\min (\mathscr{T})=\min \left(\sum_{i=0}^{k} t_{i}\right)
$$

(2) Data collection reliability $(Q)$ : the data collection reliability refers to the probability that all the data transmitted from nodes in all tiers reach the sink node without loss after a data collection cycle is completed. In the application of periodic data collection WSNs, no data loss will affect the result. However, using data aggregation increases the amount 
of useful data contained in a packet. Therefore, the probability of packet loss affecting the final result increases. Obviously, improving the reliability of data collection is very important. The greater reliability $(\mathbb{Q})$ of data collection, the better:

$$
\max (Q)=\max \{P(\text { all data transmit to sink node })\}
$$

(3) Network lifetime $(\mathscr{L})$ : the lifetime of the network refers to the time when all nodes in the network normally perform the function. Since the WSN is an ad hoc network, the death of any node in the network will affect the performance of the network. Therefore, the network lifetime is generally defined as the lifetime of the first dead node in the network. Since a periodic data collection network is adopted in this paper, the network lifetime can be represented by the data collection cycles of the network until the first node dies. Obviously, the longer the life of the network, the better. Let $E_{c}$ denote the energy consumption of a node that consumes the highest energy in the network during a data collection cycle, and $E_{\text {init }}$ denote the initial energy of the node. The network lifetime can be expressed as follows:

$$
\max (\mathscr{L})=\max \left(\frac{E_{\text {init }}}{E_{c}}\right)
$$

To sum up, the goal we want to achieve can be expressed by the following expressions:

$$
\begin{array}{ll}
\min & (\mathscr{T})=\min \left(\sum_{i=0}^{k} t_{i}\right) \\
\max & (\mathscr{Q}) \\
& =\max \{\mathrm{P}(\text { all data transmit to sink node })\} \\
\max & (\mathscr{L})=\max \left(\frac{E_{\text {init }}}{E_{c}}\right) \\
\text { s.t. } & \mathbb{Q} \geq \mathbb{Q}_{\text {std }}, \mathscr{T} \leq \mathscr{T}_{\text {std }}
\end{array}
$$

In (9), $\mathbb{Q}_{s t d}, \mathscr{T}_{s t d}$ represent the minimum standard required by the user. The goal of (9) is to improve the performance as much as possible, while ensuring that $\mathscr{T}$ and $\mathbb{Q}$ reach the minimum standard, while maximizing the network lifetime as much as possible.

\section{The Design of ATRTC Scheme}

4.1. Research Motivation. There are two main aspects of motivation for this paper: On the one hand, if the transmission power of node can be increased, the transmission range of the node will extend and the PRR does not decrease. On the other hand, in the wireless sensor network under the traditional scheme, the energy consumption of a small part of the nodes near the sink node (near-sink nodes) is much greater than that of the most of the nodes far from the sink node. This leads to the energy remaining of most nodes after the death of the network.

If transmission power of a node can be increased, then the transmission range of node can be extended and PRR

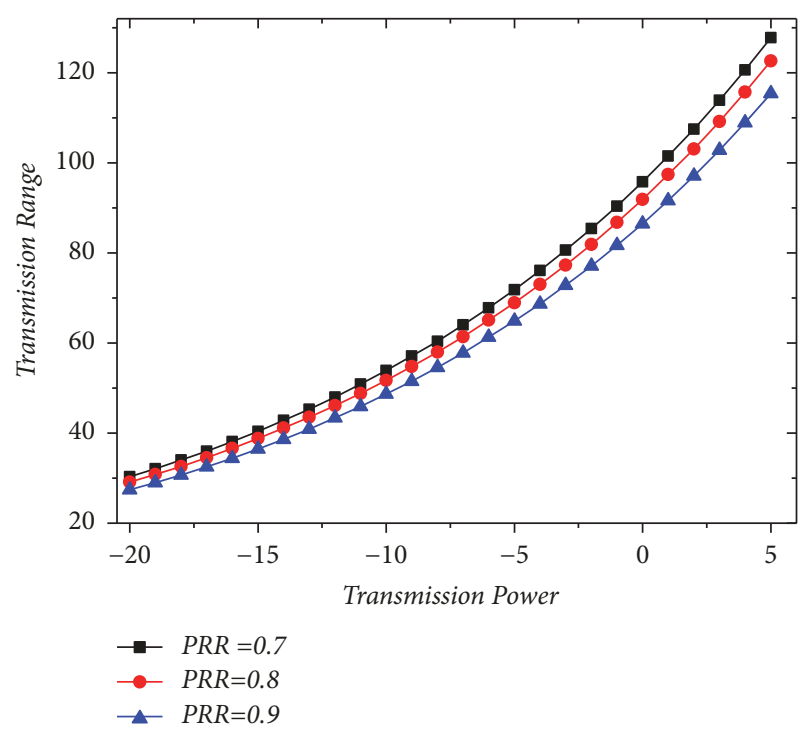

FIgURE 3: Transmission range with transmission power.

will not decrease at the same time. If the transmission range of the node can be extended to a certain level, the number of hops that node transmit data to sink can be reduced. That means the data reaching sink node need less forwarding times than before which can reduce the transmission time. Since the wireless channel between nodes is lossy, there is a possibility of packet loss during data transmission. During the transmission of a data packet from a node to the sink node, the probability of packet loss increases with each hop. Since extending the transmission range of node will reduce the number of hops, the possibility of packet loss will be reduced during the transmission to the sink node. From the perspective of the entire network, extending the transmission range improves the data collection reliability. Unfortunately, increasing the transmission power will increase energy consumption and shorten the lifetime of the node. It is unwise to increase the transmission power blindly.

In general, there are different power levels that can be adjusted in a wireless sensor node; for example, MICA2 has 26 transmit power levels $(-20 \mathrm{dBm}$ to $5 \mathrm{dBm})$. According to the reliability model in Section 3.2, the relationship between transmission range and transmission power under different PRR can be obtained. In Figure 3, the higher the transmission power, the large the transmission range, under the situation of the same PRR. And at the same transmission power, the higher the PRR, the smaller the transmission range. Assume that the network used in this paper requires the PRR to be 0.868. The maximum transmission range that can be achieved under different power levels is summarized in Table 2.

As shown in Table 2, when the transmission power is $20 \mathrm{dBm}$, the transmission range is $28 \mathrm{~m}$. Because $-20 \mathrm{dBm}$ is the minimum transmission power of the node, $28 \mathrm{~m}$ is the minimum transmission range that guarantees $\mathrm{PRR}=0.868$. When the transmission power is $-8 \mathrm{dBm}$, the transmission range of the node can reach $55.9 \mathrm{~m}$, which is about twice the minimum transmission range. And when the transmission 
TABLE 2: The transmission range under PRR $=0.868$.

\begin{tabular}{lc}
\hline Transmission power & Range \\
\hline$-20 \mathrm{dBm}(0.0100 \mathrm{~mW})$ & $28.0 \mathrm{~m}$ \\
$-19 \mathrm{dBm}(0.0126 \mathrm{~mW})$ & $29.7 \mathrm{~m}$ \\
$-18 \mathrm{dBm}(0.0158 \mathrm{~mW})$ & $31.4 \mathrm{~m}$ \\
$-17 \mathrm{dBm}(0.0200 \mathrm{~mW})$ & $33.3 \mathrm{~m}$ \\
$-16 \mathrm{dBm}(0.0251 \mathrm{~mW})$ & $35.3 \mathrm{~m}$ \\
$-15 \mathrm{dBm}(0.0316 \mathrm{~mW})$ & $37.4 \mathrm{~m}$ \\
$-14 \mathrm{dBm}(0.0398 \mathrm{~mW})$ & $39.6 \mathrm{~m}$ \\
$-13 \mathrm{dBm}(0.0501 \mathrm{~mW})$ & $41.9 \mathrm{~m}$ \\
$-12 \mathrm{dBm}(0.0631 \mathrm{~mW})$ & $44.4 \mathrm{~m}$ \\
$-11 \mathrm{dBm}(0.0794 \mathrm{~mW})$ & $47 \mathrm{~m}$ \\
$-10 \mathrm{dBm}(0.1000 \mathrm{~mW})$ & $49.8 \mathrm{~m}$ \\
$-9 \mathrm{dBm}(0.1259 \mathrm{~mW})$ & $52.8 \mathrm{~m}$ \\
$-8 \mathrm{dBm}(0.1585 \mathrm{~mW})$ & $55.9 \mathrm{~m}$ \\
$-7 \mathrm{dBm}(0.1995 \mathrm{~mW})$ & $59.2 \mathrm{~m}$ \\
$-6 \mathrm{dBm}(0.2512 \mathrm{~mW})$ & $62.7 \mathrm{~m}$ \\
$-5 \mathrm{dBm}(0.3162 \mathrm{~mW})$ & $66.4 \mathrm{~m}$ \\
$-4 \mathrm{dBm}(0.3981 \mathrm{~mW})$ & $70.3 \mathrm{~m}$ \\
$-3 \mathrm{dBm}(0.5012 \mathrm{~mW})$ & $74.5 \mathrm{~m}$ \\
$-2 \mathrm{dBm}(0.6310 \mathrm{~mW})$ & $78.9 \mathrm{~m}$ \\
$-1 \mathrm{dBm}(0.7943 \mathrm{~mW})$ & $83.6 \mathrm{~m}$ \\
$0 \mathrm{dBm}(1.0000 \mathrm{~mW})$ & $88.6 \mathrm{~m}$ \\
$1 \mathrm{dBm}(1.2589 \mathrm{~mW})$ & $93.8 \mathrm{~m}$ \\
$2 \mathrm{dBm}(1.5849 \mathrm{~mW})$ & $99.4 \mathrm{~m}$ \\
$3 \mathrm{dBm}(1.9953 \mathrm{~mW})$ & $105.2 \mathrm{~m}$ \\
$4 \mathrm{dBm}(2.5119 \mathrm{~mW})$ & $111.5 \mathrm{~m}$ \\
$5 \mathrm{dBm}(3.1623 \mathrm{~mW})$ & $118.1 \mathrm{~m}$ \\
\hline &
\end{tabular}

power is $5 \mathrm{dBm}$, the range can reach $118.1 \mathrm{~m}$, which can reach more than 4 times the minimum range.

(2) Under the traditional Constant Transmission Power and Range (CTPR) scheme, there is a big difference in energy consumption of nodes in different locations in the network. Because the nodes close to sink node need to bear forwarding a large amount of data sent from far-sink nodes, the energy consumption of near-sink node is much great than that of farsink node. Because of the death of one node in the wireless sensor network, it will have a great impact on the network, so that the entire network cannot complete the predetermined function. So, once a node dies, it is regarded that the entire network is dead. At this time, far-sink nodes still have a lot of energy left. In other words, there is still a large amount of energy in the network that can be used after the network died.

We analyzed a network consisting of 500 wireless sensor nodes and a sink node using traditional Constant Transmission Power and Range (CTPR) scheme. Some specific parameters of this network are summarized in Table 3.

We have calculated the energy consumption of each node of the network in one data collection cycle and the remaining energy of each node after the network died. As shown in Figure 4, the energy consumption decreases from the nearsink node to the far-sink node under the CTPR scheme. The trend of energy consumption for receiving and transmitting is

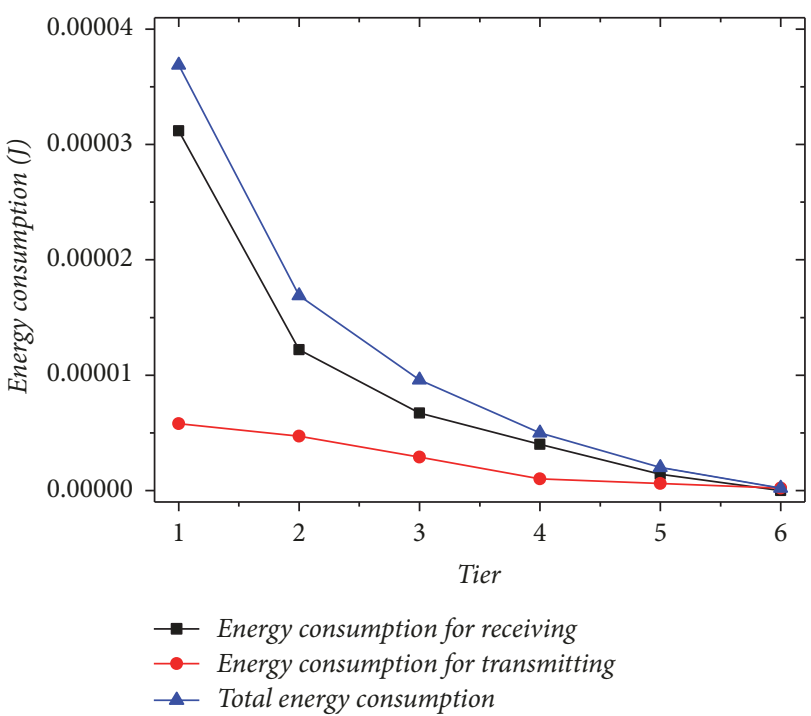

FIGURE 4: Energy consumption in one cycle.

TABLe 3: Parameters of network.

\begin{tabular}{lcc}
\hline Symbol & Description & Value \\
\hline$N$ & total number of node in network & 500 \\
$R$ & radius of network & $126 \mathrm{~m}$ \\
$d$ & width of tier & $21 \mathrm{~m}$ \\
$\varepsilon$ & aggregation ratio & 0.3 \\
$r$ & initial transmission range of node & $28 \mathrm{~m}$ \\
$P^{\text {send }}$ & initial transmission power of node & $-20 \mathrm{dBm}$ \\
$P^{\text {receive }}$ & receiving power of node & $-20 \mathrm{dBm}$ \\
\hline
\end{tabular}

similar to the trend of total energy consumption. The energy consumption of the node in tier 1 is the largest. The total energy consumption and energy consumption for receiving of nodes in tier 1 are greater than twice that of nodes in tier 2 . Since nodes in tier 6 are located at the outermost tier of the network, there is no child node and no energy consumption for receiving data, and their total energy consumption and energy consumption of the transmitting are also minimum. The total energy consumption of the first-level nodes is as high as 219 times the energy consumption of nodes in tier 6 . We can see the huge difference between them.

Figure 5 shows the lifetime and the residual energy of nodes at each tier after the network died under the CTPR scheme. The black line in the figure shows the lifetime of the node, and the cyan line shows the residual energy after the network died. First, from the black line, it can be seen that the lifetime of the nodes from the first layer to the sixth layer is longer and longer. Since the lifetime of the network is equal to the lifetime of the first dead node, under the CTPR scheme, the lifetime of the network is equal to the lifetime of the node in tier 1 . Second, from the cyan line, it can be seen that the residual energy of the node in tier 1 is close to 0 , while the residual energy of nodes in other tiers reaches $60 \%$ of the total energy.

From the analysis in Figures 4 and 5, we can see that the energy utilization rate of the traditional network using 


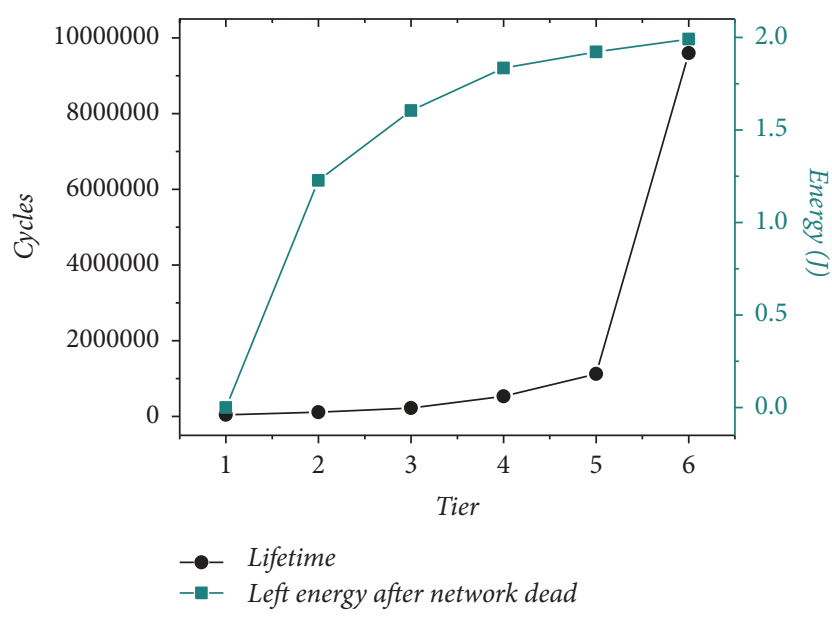

Figure 5: Lifetime and left energy of network.

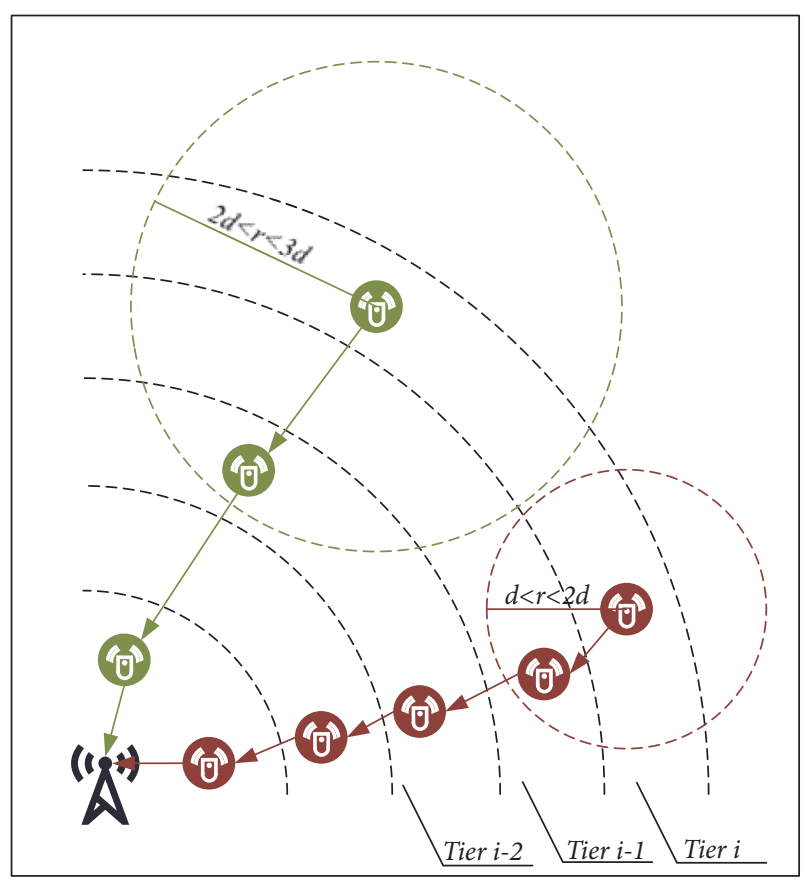

ATRTC Scheme

(0) CTPR Scheme

FIGURE 6: ATRTC scheme versus CTPR scheme.

CTPR scheme is very low, and the energy consumption of the network is uneven. After the network died, most of the nodes in the network have a large amount of residual energy. In one word, the CTPR scheme needs to be improved.

Based on the above two points, there is a scheme that can utilize the remaining energy of the far-sink nodes to increase the transmission power of the nodes and improve the network performance. By analyzing the network energy consumption and performance, we propose an Adaptive Transmission Range Based Topology Control (ATRTC) scheme. It can improve the reliability of data collection and reduce the delay of data collection without affecting the network lifetime.

4.2. The ATRTC Scheme. The main idea of the ATRTC scheme is to extend the transmission range of the nodes which have remaining energy by increasing the transmission power of the nodes, so that it can reduce the network collection time and improve the reliability of the network collection. As shown in Figure 6, when the network uses the CTPR scheme, the transmission range $r$ of the node is in the range of $d<r<2 d$. This transmission range can make sure that the node can transmit data to the parent node in the next tier. While the ATRTC scheme increases the transmission distance of the node by increasing the transmission power of the node to a range of $2 d<r<3 d$, so that the node in tier $i$ can transmit the data to node both the tier $i-1$ and tier $i$ - 2 nodes. By modifying the transmission protocol, nodes in tier $i$ can transmit data only to the nodes in tier $i-2$. We call this type of transmission a cross-tier transmission. The ATRTC scheme adaptively extends the transmission range and makes sure PRR reach a level that user required according to the condition of the energy remaining of nodes in different tiers. Through the cross-tier transmission, the number of hops transmitted from the node to the sink node is reduced. In this way, the reliability of the network data collection can be improved and the network data collection delay can be reduced, so that the network performance can be improved.

According to the main idea of the ATRTC scheme, we have designed the ATRTC scheme algorithm (see Algorithm 1).

4.3. The Calculation of Energy Consumption. Calculating energy consumption in the network is very important for the ATRTC scheme. ATRTC scheme needs to adjust the transmission power of node in each tier according to the energy consumption in the network, since the biggest part of the energy consumption of the nodes is the data transmission and the energy consumed by other aspects is negligible. Therefore, the energy consumption of a node is approximately equal to the energy consumption of a node for data transmission. The energy consumption of a node needs to be calculated based on the amount of data transmitted by the node data.

The data transmission of a node includes two parts: receiving data from the child nodes and sending data to the parent nodes. Since broadcast mechanism is adopted in the network, a node can have more than one parent node at the same time. That means the data sent by a node can be received by multiple parent nodes simultaneously. And that also means different parent nodes may have the same child node. It is more complicated than the unicast mechanism where just one node sends data to another node. In the same tier of the network, if the position of nodes is different, the number of their parent nodes is also different. In order to simplify the calculation, we have studied the positions of the most and the least number of parent nodes in a tier and given the calculation methods respectively shown below. 


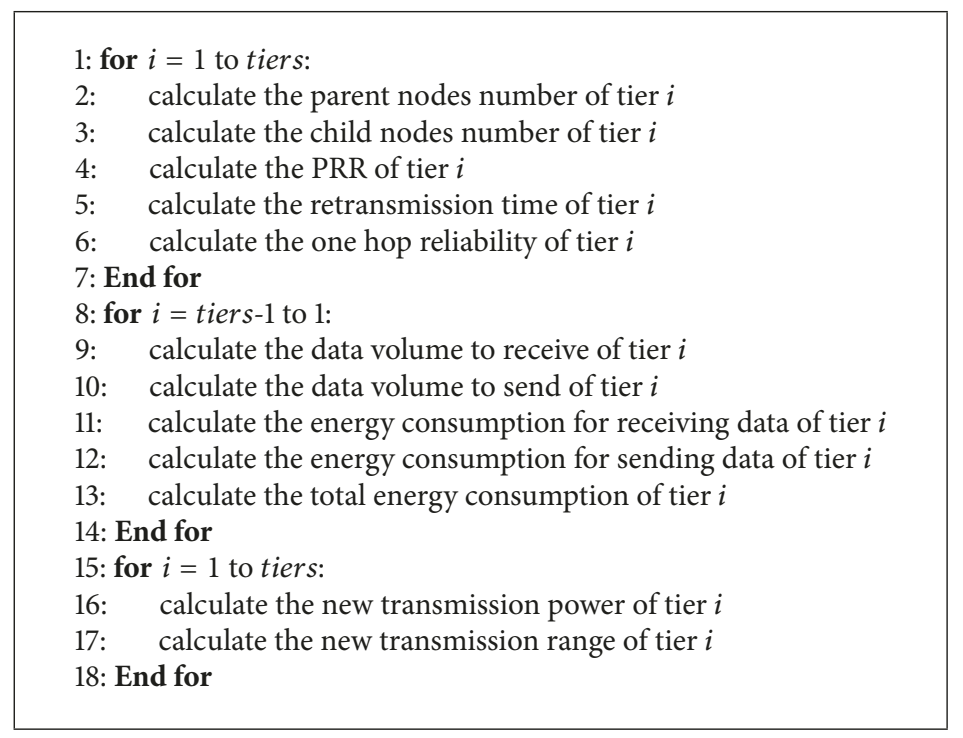

Algorithm 1: ATRTC Scheme Algorithm.

Theorem 1. The node $v$ in the outermost of the tier $i$ covers the minimum area of tier where the parent nodes are located. The area is

$$
\begin{aligned}
S_{\text {min }}= & r_{1}^{2} \cdot(\arccos \angle A C B-\sin \angle A C B \cdot \cos \angle A C B) \\
& +r_{2}^{2} \cdot(\arccos \angle C A B-\sin \angle C A B \cdot \cos \angle C A B)
\end{aligned}
$$

where

$$
\begin{aligned}
& \cos \angle C A B=\frac{l^{2}+r_{2}^{2}-r_{1}^{2}}{2 \cdot r_{1} \cdot r_{2}}, \\
& \cos \angle A C B=\frac{r_{1}^{2}+l^{2}-r_{2}^{2}}{2 \cdot r_{1} \cdot l}
\end{aligned}
$$

Proof. As shown in Figure 7, the position indicated by point $\mathrm{C}$ is the position of the sink node. Point $\mathrm{A}$ is the position of the node $v$ which has the least number of parent nodes. All the parent nodes of node $v$ are located in the shadow area. The shadow area can be seen as the area intersected by the circle that center is $\mathrm{C}$ and radius is $r_{1}$ and the circle that center is $\mathrm{A}$ and radius is $r_{2}$. Obviously, the longer the distance between two centers, the smaller the area of intersection. And the position of point A leads to the longest distance of two centers, so node $v$ has the least number of parent nodes.

The three sides $r_{1}, r_{2}$, and $l$ of triangle $\mathrm{ABC}$ are known, and the cosine value of $\angle C A B$ and $\angle A C B$ can be calculated by cosine theorem. And the area of sector $\mathrm{DAB}$ and sector $\mathrm{CAB}$ can be calculated by using sector area formula. And the area of isosceles triangle $\mathrm{DAB}$ and isosceles triangle $\mathrm{CAB}$ can also calculated by using $r_{1}, r_{2}$ and cosine value of $\angle \mathrm{CAB}$ and $\angle A C B$. Finally, $S_{\text {shadow area }}=\left(S_{\text {sector } D A B}-S_{\text {triangle } D A B}\right)+$ $\left(S_{\text {sector } C A B}-S_{\text {triangle } C A B}\right)$.

Theorem 2. The node $v$ in the innermost of the tier $i$ covers the maximum area of tier where the parent nodes are located. The area is

$$
S_{\max }=S_{1}-S_{2}
$$

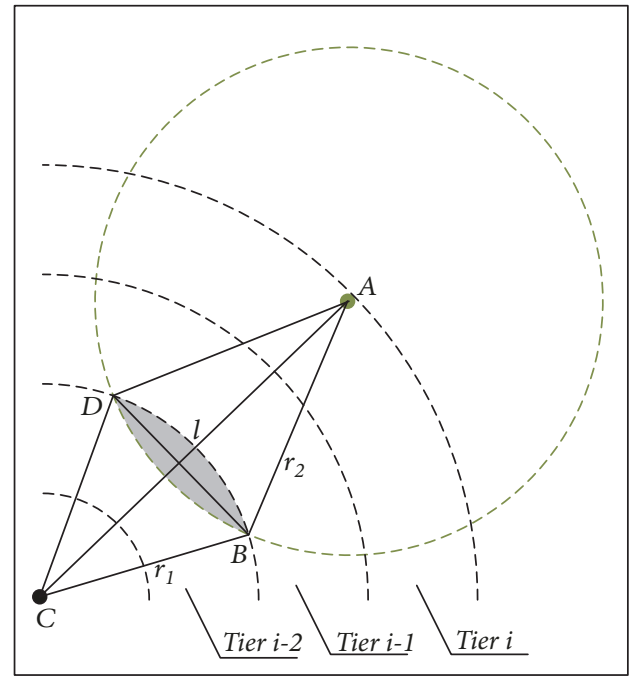

- Node $v$ in outermost position of tier $i$

- Sink Node

FIGURE 7: Min area that node covered the parent tier.

Proof. As shown in Figure 8, $S$ is the shadow area and $S_{1}$ is the sum of the areas of $S$ and $S_{2}$. Because the shape of $S$ is irregular, it cannot be obtained directly. Fortunately, the area of $S_{1}$ can be seen as node $v$ in the outermost of tier $i-1$ and its parent nodes in tier $i-2$. Then the area of $S_{1}$ can be calculated by Theorem 1 . And the area of $S_{2}$ can be seen as node $v$ in the outermost of tier $i-1$ and its parent nodes in tier $i-3$, so the area of $S_{2}$ can also be calculated by Theorem 1 .

Figures 9 and 10 illustrate the minimum area and maximum area of each tier under different transmission range, respectively, in the network (the parameters of network are listed in Table 3). For the same transmission range, the larger the tier number of nodes, the larger the area covered. And it 
can be seen that the increase of the coverage area decreases as the tier number increases. For different transmission ranges, the greater the transmission range at the same tier number, the greater the area covered.
Theorem 3. In the circle network that the radius is, $N$ nodes are uniformly deployed. If area $S$ in the network is given, the number of node $n$ in the area is

$$
n=S \cdot \rho=\frac{S \cdot N}{\pi \cdot R^{2}} \quad \text { where } N \text { is the total number of nodes in the network, } R \text { is the radius of the network }
$$

Proof. Because $N$ nodes are uniformly deployed in the network, the density of nodes in different area of the network is the same. $N$ and $R$ are given, and the density of node can be calculated: $\rho=N /\left(\pi \cdot R^{2}\right)$. And the area $S$ is given, so the number of nodes in the area is $n=(S \cdot N) /\left(\pi \cdot R^{2}\right)$.

The minimum number of parent nodes of nodes in tier $i$ can be calculated by using Theorems 1 and 3, and the maximum number of parent nodes can be calculated by using Theorems 2 and 3.

In order to calculate the receiving data volume of a node, the number of child nodes and the sending data volume of its child nodes need to be calculated at first. The methods to calculate the number of child nodes are given below.

Theorem 4. Assume that node $v$ in tier $i$ has child nodes in tier $j(i<j)$. If the number of parent nodes of a node in tier $j$ $\left(n_{j}^{\text {parent }}\right)$ and the total number of nodes in tier $j$ and tier $i\left(n_{j}\right.$ and $n_{i}$ ) are known, the average number of child nodes of node $v$ can be calculated by the following equation:

$$
\mathrm{n}_{i}^{\text {child }}=\frac{n_{j}^{\text {parent }} \cdot n_{j}}{n_{i}}
$$

Proof. If a node in tier $j$ has only one parent node in tier $i$, each node in tier $i$ receives data from average $n_{j} / n_{i}$ child nodes in tier $j$. If broadcasting is used, i.e., a node in tier $j$ has multiple parent nodes in tier $i$, and each node in tier $i$ receives data from average $n_{j}^{\text {parent }} \cdot n_{j} / n_{i}$ child nodes in tier $j$.

The number of child nodes can be obtained by using Theorem 4, and we also need to obtain the sending data volume of child nodes for calculating the receiving data volume of parent node. And the sending data volume of child node needs to be obtained by receiving data volume of child node. The sending and receiving data volume of nodes in the outermost tier is known. So, the receiving and sending data volume can be calculated from the outer tier to the inner tier. Theorem 5 gives the calculation method of the receiving data volume of the node. Theorem 6 gives the calculation method of sending data volume of the node.

Theorem 5. Assume that node $v$ in tier $i$ has child nodes in tier $j(i<j)$. In a data collection cycle, the sending data volume of child nodes in tier $j\left(C_{j}^{\text {send }}\right)$ and the number of child nodes of node in tier $i\left(n_{i}^{\text {child }}\right)$ are known. If the PRR of data transmission from child node to node $v$ is $p$, and the retransmission time is $\delta$, then the receiving data volume of node $v$ is

$$
C_{i}^{\text {receive }}=n_{i}^{\text {child }} \cdot C_{j}^{\text {send }} \cdot\left[1-(1-p)^{1+\delta}\right]
$$

Proof. If the PRR is $p$ and retransmission times is $\delta$, the possibility of a successful data transmission is $1-(1-p)^{1+\delta}$. If the wireless channel is lossless, the receiving data volume of node $v$ is $n_{i}^{\text {child }} \cdot C_{j}^{\text {send }}$. So, under the lossy wireless channel, the receiving data volume of node $v$ can be calculated; that is, $n_{i}^{\text {child }} \cdot C_{j}^{\text {send }} \cdot\left[1-(1-p)^{1+\delta}\right]$.

Theorem 6. In a data collection cycle, if the retransmission times $\delta$, the receiving data volume $C_{i}^{\text {receive }}$, and the aggregate function $f($ data) are known, the sending data volume of node $v$ in tier $i$ is

$$
C_{i}^{\text {send }}=(\delta+1) \cdot f\left(C_{i}^{\text {receive }}+1\right)
$$

Proof. The sending data of node $v$ are generated by aggregate function. And the input data of aggregate function are comprised of the data generated by self (size of 1 data packet) and the data received from its child nodes. The data volume of input data is $C_{i}^{\text {receive }}+1$. If the aggregate function $f($ data $)$ is known, the output data volume is $f\left(C_{i}^{\text {receive }}+1\right)$. If node $v$ retransmits data $\delta$ times, the sending data volume is $(\delta+1)$. $f\left(C_{i}^{\text {receive }}+1\right)$

The sending and receiving data volume of nodes in each tier can be calculated by using Theorems 5 and 6 . They are the essential values to calculate the energy consumption of nodes in each tier. And the calculation methods of energy consumption according to data volume are shown in theorem below.

Theorem 7. Assume the following values are known: sending data volume $C_{i}^{\text {send }}$ and receiving data volume $C_{i}^{\text {receive }}$ of a node in tier $i$, the power for sending data $P_{i}^{\text {send }}$ and receiving data $P_{i}^{\text {receive }}$, the sending rate $V_{\text {send }}$, and receiving rate $V_{i}^{\text {receive }}$. The energy consumption of a node for sending data in a data collection cycle is

$$
E_{\text {send }}=\frac{C_{i}^{\text {send }} \cdot P_{i}^{\text {send }}}{V_{\text {send }}}
$$




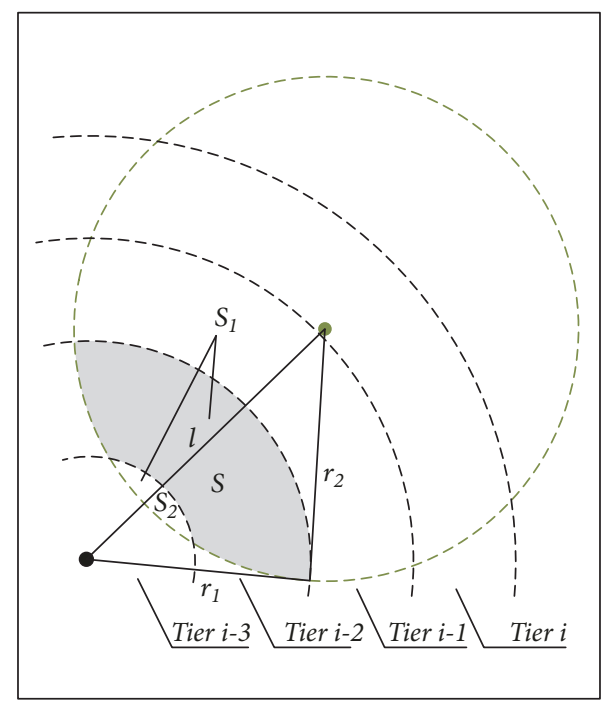

Node in innermost position of tier $i$

Sink Node

FIgure 8: Max area that node covered the parent tier.

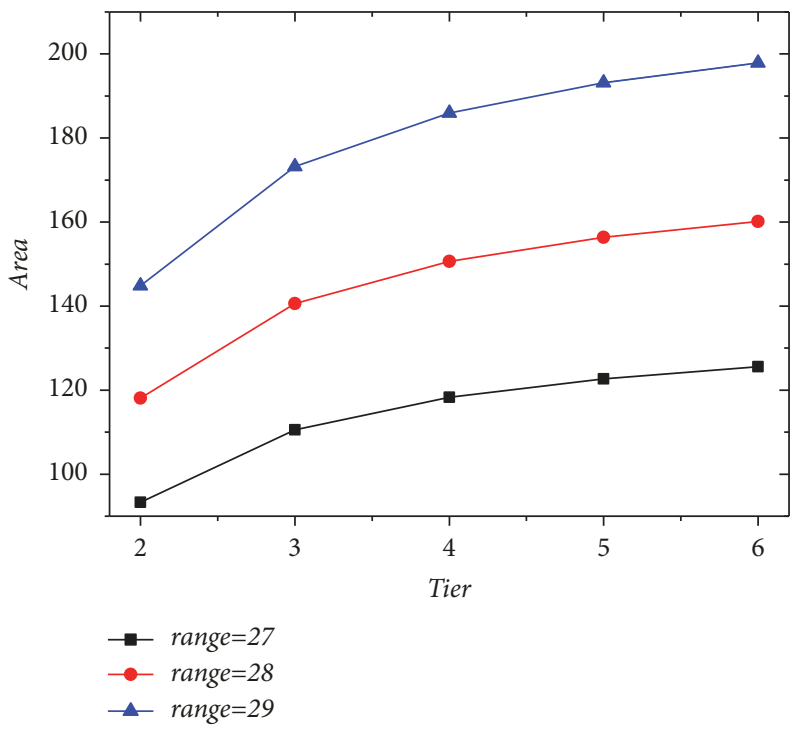

FIgURE 9: Min covered area with ranges.

The energy consumption of a node for receiving data in a data collection cycle is

$$
E_{\text {receive }}=\frac{C_{i}^{\text {receive }} \cdot P_{i}^{\text {receive }}}{V_{\text {receive }}}
$$

The total energy consumption of a node in a data collection cycle is

$$
E_{\text {total }}=E_{\text {send }}+E_{\text {receive }}
$$

Proof. If a power $P$ and time $t$ are known the energy consumption $E$ can be calculated as $E=P \cdot t$. If the data volume

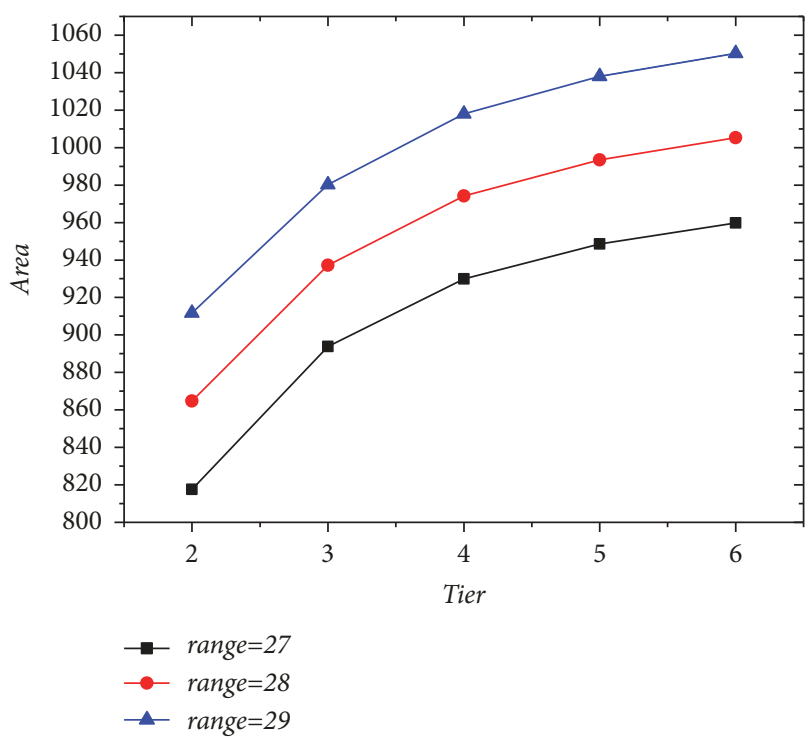

Figure 10: Max covered area with ranges.

$C$ and transmission rate $V$ are known, the time $t$ can be calculated as $t=C / V$. So, $E=C \cdot P / V$. To calculate the energy consumption for sending data, just substitute $C_{i}^{\text {send }}$, $P_{i}^{\text {send }}$, and the $V_{\text {send }}$ into the equation $E=C \cdot P / V$, that is, $E_{\text {send }}=C_{i}^{\text {send }} \cdot P_{i}^{\text {send }} / V_{\text {send }}$ And the energy consumption for receiving data can be calculated by using the method; that is, $E_{\text {receive }}=C_{i}^{\text {receive }} \cdot P_{i}^{\text {receive }} / V_{\text {receive }}$. Since the energy of a node is mostly used by sending data and receiving data. Therefore, the total energy consumption of the node is $E_{\text {total }}=$ $E_{\text {send }}+E_{\text {receive }}$.

4.4. The Calculation of Transmission Range. The key point of the ATRTC scheme is to extend the transmission range properly, and the PRR should not be decreased at same time. To achieve this goal, the transmission power needs to be increased.

Theorem 8. Assume the following values are known: the energy consumption of node $v$ in tier $i$ for receiving data $E_{i}^{\text {receive }}$, the sending data volume of node $v C_{i}^{\text {send }}$, the sending rate $V_{\text {send }}$, the maximum energy consumption in the network (usually the node in tier 1) $E_{\max }$, and the max transmission power that the node can adjust determined by hardware $P_{\text {limit }}$. Under the ATRTC scheme, the transmission power of a node can adjust is

$$
P_{i}^{\text {send }}=\min \left\{P_{\text {limit }}, \frac{\left(E_{\max }-E_{i}^{\text {receive }}\right) \cdot V_{\text {send }}}{C_{i}^{\text {send }}}\right\}
$$

Proof. Under the ATRTC scheme, some nodes can increase the transmission power by using the residual energy. The energy which can be used for sending data is $E_{\max }-E_{i}^{\text {receive }}$. According to the equation $E=C \cdot P / V$ in proof of Theorem 7, the power can be calculated as $P=E \cdot V / C$. Since the energy can use $E_{\max }-E_{i}^{\text {receive }}$, and the sending rate $V_{\text {send }}$ and the sending data volume are known, the new transmission power of node under ATRTC scheme can be 
calculated: $\left(E_{\max }-E_{i}^{\text {receive }}\right) \cdot V_{\text {send }} / C_{i}^{\text {send }}$. And the hardware limit of the transmission power needs to be considered; if the results calculated by the equation are beyond the $E_{\text {limit }}$, the transmission power should be $E_{\text {limit }}$. So, the new transmission power under ATRTC scheme can be expressed as $\min \left\{E_{\text {limit }}, P_{i}^{\text {send }} \cdot\left(E_{\max }-E_{i}^{\text {receive }}\right) / E_{i}^{\text {send }}\right\}$.

Theorem 9. If the transmission power $P_{s}$ and the PRR standard $p$ are known, the max transmission range can be calculated by the following equation:

$$
r_{\max }=\frac{\left(10^{\left[P L(r)-P L\left(r_{0}\right)\right] / 10 n}\right)}{r_{0}}
$$

where

$$
P L(r)=P_{s}+1.28 \ln \left[2\left(1-p^{1 / 8 f}\right)\right]-P_{n}
$$

Proof. According to the reliability model in Section 3.2, the relationship of PRR, transmission power, and transmission range can be known by (2), (3), and (4). And according to (2), the relationship between signal noise ratio $\gamma$ and PRR $p$ can be known: $\gamma=-1.28 \ln \left[2\left(1-p^{1 / 8 f}\right)\right](2)$ '. According to (3), the relationship of $P L(r)$ and $\gamma$ can be known: $P L(r)=$ $P_{t}-\gamma-P_{n}(3)^{\prime}$. And according to (4), the relationship between $r$ and $P L(r)$ can be known: $r=\left(10^{\left[P L(r)-P L\left(r_{0}\right)\right] / 10 n}\right) / r_{0}(4)$ '. Substituting (2)' to (3)', and then substituting (3)' to (4)', we can get the relationship between the transmission range $r$, the transmission power $P_{s}$, and the PRR $p$.

The max transmission range of node under a certain transmission power and a certain PRR can be calculated by using Theorem 9. But this is not a proper transmission range, because the excessive transmission range will lead to the data redundancy and energy waste. And the data collection time will be increased accordingly. Therefore, after calculating the max transmission range, the new transmission range needs to be adjusted based on the max transmission range. In ATRTC scheme, we suggest that the proper transmission range should satisfy the condition of not more than $3 d$ in length and the minimum number of parent nodes is 2 .

Under the ATRTC scheme, a node has residual energy that can achieve cross-tier transmission. In order to figure out which tier the node can reach, we show the method in Theorem 10.

Theorem 10. If the transmission range $r$ of node $v$ in tier $i$ and the width of tier $d$ are known, the tier number of the tier that node $v$ can reach is

$$
\text { tier }=i-\left\lfloor\frac{r}{d}\right\rfloor
$$

Proof. If the transmission range $r$ and the width of tier $d$ are known, the number of tiers that node $v$ can completely span can be calculated, that is, $\lfloor r / d\rfloor$. Because the order of tier number from the outermost tier to the sink is descending, and the tier number of node $v$ is also known, the tier number of node $v$ can be $i-\lfloor r / d\rfloor$

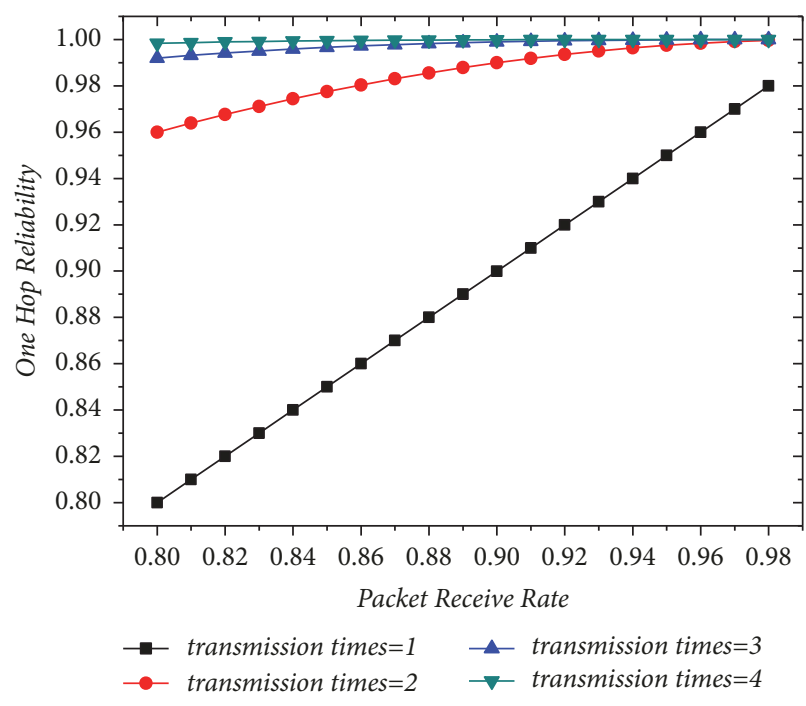

FIgURE 11: One-hop reliability with transmission times.

4.5. The Calculation of Data Collection Reliability. Due to the data aggregation technology used in the network, the amount of information in a single packet increases, the final result generated after data collection is more vulnerable and affected by packet loss. Therefore, improving the reliability of data collection helps to ensure the accuracy of data collection. Theorem 11 gives the calculation method of the reliability of the node to transmit data to the parent node. Theorem 12 gives the calculation method for the reliability of the data sent by node reach to the sink node successfully. And Theorem 13 gives the calculation method of network data collection reliability.

Theorem 11. The one-hop reliability of node in tier $i$ is defined as the possibility of data sent by node in tier i reach to the parent nodes successfully by using broadcast and retransmission mechanism. If the minimum numbers of parent nodes $x$, the $\operatorname{PRR} p$, and the retransmission time $\delta$ are known, the one-hop reliability of node in tier $i$ is

$$
\varphi_{i}=1-(1-p)^{x \cdot(1+\delta)}
$$

Proof. If the PRR of the data transmission is $p$, the possibility of packet loss is $(1-p)$. If the node transmits the same data packet $m$ times, the possibility of packet loss is $(1-p)^{m}$. The $\delta$ times retransmission can be seen as $\delta+1$ times data transmission, the possibility of packet loss is $(1-p)^{1+\delta}$. And transmitting data to $x$ parent nodes simultaneously can be seen as $x$ times data transmission, the possibility of packet loss is $(1-p)^{x}$. If node transmits data packet to parent nodes by using broadcast and retransmission mechanism, the possibility of packet loss is $(1-p)^{x \cdot(1+\delta)}$. Therefore, the possibility of successful data transmission, i.e., the one-hop reliability, is $1-(1-p)^{x \cdot(1+\delta)}$.

According to the proof of Theorem 11, the data transmission by broadcast retransmission mechanism can be seen as multiple transmissions of the same data packet. Figure 11 
shows the relationship between one-hop reliability and PRR under different transmission times. As shown in Figure 11, first, the greater the one-hop reliability the greater the PRR under the same transmission times. Second, the more transmission times, the greater one-hop reliability under the same PRR. When the transmission times $=1$, the one-hop reliability of node is equal to the PRR. When transmission time $=2$, the one-hop reliability of node is greatly improved compared to that when transmission time $=1$. Even when the $P R R=0.8$, the one-hop reliability can reach 0.96 under transmission time $=2$. It can be seen that when the PRR is relatively low, using broadcast and retransmission mechanism can significantly improve the one-hop reliability.

Theorem 12. Assume that a data packet from a node reaches sink node successfully needs multiple hop transmission. If the one-hop reliability of each hop is $\varphi_{1}, \varphi_{2}, \cdots, \varphi_{m}$, the reliability of a data transmission from the node in tier $i$ to the sink node is

$$
\Phi_{i}=\prod_{k=1}^{m} \varphi_{k}
$$

Proof. The data sent by the node in tier $i$ needs to forward by the parent node closer to the sink node. The reliability of successful transmission to the sink node is based on the reliability of each one-hop reliability of node to forward the data. Therefore, the reliability of data transmission from the node in tier $i$ to the sink node can be expressed as $\Phi_{i}=$ $\prod_{k=1}^{m} \varphi_{k}$

Theorem 13. If the reliabilities of data transmission from node in each tire to the sink node $\Phi_{1}, \Phi_{2}, \cdots, \Phi_{m}$ are known, the reliability of network data collection $\mathbb{Q}$ is

$$
\mathcal{Q}=\min \left\{\Phi_{1}, \Phi_{2}, \cdots, \Phi_{m}\right\}
$$

Proof. The distances between the nodes in difference positions and the sinks are different, the reliability of the transmission to the sink is also different. The minimum reliability is used to measure the reliability of network data collection. It can make sure the reliability of the transmission of data sent from any node reach to the sink node is not less than the minimum reliability $\mathbb{Q}$.

4.6. The Calculation of Data Collection Time. The data collection time in this paper is the time length from the beginning to the finish of data collection in the network. According to the data collection model in Section 3.3, the parent nodes need to wait until the child nodes finish the data transmission. Under the ATRTC scheme, the transmission ranges of nodes in each tier are different. The node that has residual energy can achieve cross-tier transmission. For example, the nodes in tier 8 can transmit data to the nodes in tier 6 rather than tier 7. And the nodes in tier 7 can transmit data to the nodes in tier 5. So that the nodes in tier 7 need not to wait the nodes in tier 8 . The nodes in tier 7 and tier 8 can transmit data to their parent nodes at the same time as long as their parent nodes are not in the same tier. So, under the ATRTC scheme, there are two paths that can transmit data to the sink node if some conditions are satisfied. The sink node usually has a stable power supply, and the data transmission function is more powerful than the sensor node. It is assumed that the sink node can receive data transmitted from nodes of two tiers at the same time.

Theorem 14. Assume the node $v$ in tier $i$ has child nodes in tier $j$. If the number of child nodes of node $v \mathrm{n}_{i}^{\text {child }}$, the average sending data volume of each child node $C_{j}^{\text {send }}$ and the sending rate $V_{\text {receive }}$ of nodes are known, and the time of data transmission from child nodes to node $v$ is

$$
t=\frac{\mathrm{n}_{i}^{\text {child }} \cdot C_{j}^{\text {send }}}{V_{\text {receive }}}
$$

Proof. Assume the sensor node usually has multiple data channels, and nodes in the same tier can transmit data simultaneously if their parent nodes are not the same. The parent nodes can receive data from only one child node at one time. If a parent node has multiple child nodes, the child nodes need to transmit data in order. The sending rate and receiving rate are the same value in this paper. If the wireless channel is lossless, the transmission time from nodes in tier $\mathrm{j}$ to their parent nodes in tier $i$ can be calculated by the receiving data volume of parent nodes. But, if the wireless channel is lossy, using the total sending data volume of child nods is more accurate. Therefore, the time of data transmission from child nodes to node $v$ can be expressed as $\mathrm{n}_{i}^{\text {child }} \cdot C_{j}^{\text {send }} / V_{\text {receive }}$.

Theorem 15. Assume that there are two paths for the data collection, and each path is comprised of nodes in $m$ different tiers. The two paths can transmit data simultaneously. The data transmission times on the first path are $t_{11}, t_{12}, \cdots, t_{1 m}$. The data transmission times on the second path are $t_{21}, t_{22}, \cdots$, $t_{2 m}$. The data collection time of the network can be calculated by the following equation:

$$
\mathscr{T}=\max \left\{\sum_{i=1}^{m} t_{1 i}, \sum_{i=1}^{m} t_{1 i}\right\}
$$

Proof. Since the parent node needs to wait for all child nodes to complete the transmission to start data transmission, the transmission time on each path is $\sum_{i=1}^{m} t_{i}$. For the two paths that can be transmitted simultaneously, the data collection time is determined by the transmission time of the last completed path. Therefore, the data collection time of the network can be expressed as $\max \left\{\sum_{i=1}^{m} t_{1 i}, \sum_{i=1}^{m} t_{1 i}\right\}$.

The data collection time of the network under ATRTC scheme can be calculated by using Theorems 14 and 15. For that under CTPR scheme, every transmission time from child tier to parent tier need to be calculated by using Theorem 14 and the data collection time of the network under CTPR scheme can be calculated by accumulating them. 


\subsection{The Calculation of Network Lifetime}

Theorem 16. If the initial energy of node $E_{\text {init }}$ and the total energy consumption $E_{c}$ in a data collection cycle are known, the lifetime of a node is

$$
\ell=\left\lfloor\frac{E_{\text {init }}}{E_{c}}\right\rfloor
$$

Proof. The lifetime of a node is defined as the number of data collection cycle from the full state to the empty state of the battery of a node. Therefore, the number of data collection cycle can be easily calculated by the known initial energy of node $E_{\text {init }}$ and energy consumption $E_{c}$ in a data collection cycle: $\left\lfloor E_{\text {init }} / E_{c}\right\rfloor$.

Theorem 17. Assume the network has $m$ tiers, and the lifetime of nodes in each tier is $\ell_{1}, \ell_{2}, \cdots, \ell_{m}$. The lifetime of the network can be expressed as follows:

$$
\mathscr{L}=\min \left\{\ell_{1}, \ell_{2}, \cdots, \ell_{m}\right\}
$$

Proof. According to the definition of the network lifetime in Section 3.4, the lifetime of the network is determined by the lifetime of the node first died. That means the lifetime of the network is the minimum lifetime of nodes in all tiers. Therefore, the lifetime of network can be expressed as $\min \left\{\ell_{1}, \ell_{2}, \cdots, \ell_{m}\right\}$.

In a data collection cycle, the data volume received and sent by nodes in different tiers is different. The data transmission time of nodes in different tiers is also different. Therefore, the lifetime expressed by using the data collection cycle is more convenient than using the transmission time. The lifetime of the network can be easily calculated by using Theorems 16 and 17.

\section{Analysis and Comparison}

In this section, the wireless sensor network under Adaptive Transmission Range Based Topology Control (ATRTC) scheme will be compared with the wireless sensor network under Constant Transmission Power and Range (CTPR) scheme. The network structure, the data collection reliability of the network, the data collection time, and so on will be compared and analyzed in the following contents.

5.1. Network Structure. Under the ATRTC scheme, the transmission power and the transmission range of nodes in each tier are different compared with these under CTPR scheme.

The transmission power of nodes under ATRTC scheme and under CTPR scheme is compared in Figure 12. As can be seen from Figure 12, the transmission power of nodes in most tiers under ATRTC scheme is increased compared with that under CTPR scheme. The transmission power of node in all tiers is the same value under CTPR scheme. And the more the residual energy of the nodes, the higher the transmit power under the ATRTC scheme.

Under the ATRTC scheme, increasing the transmission power of a node can extend the transmission range and

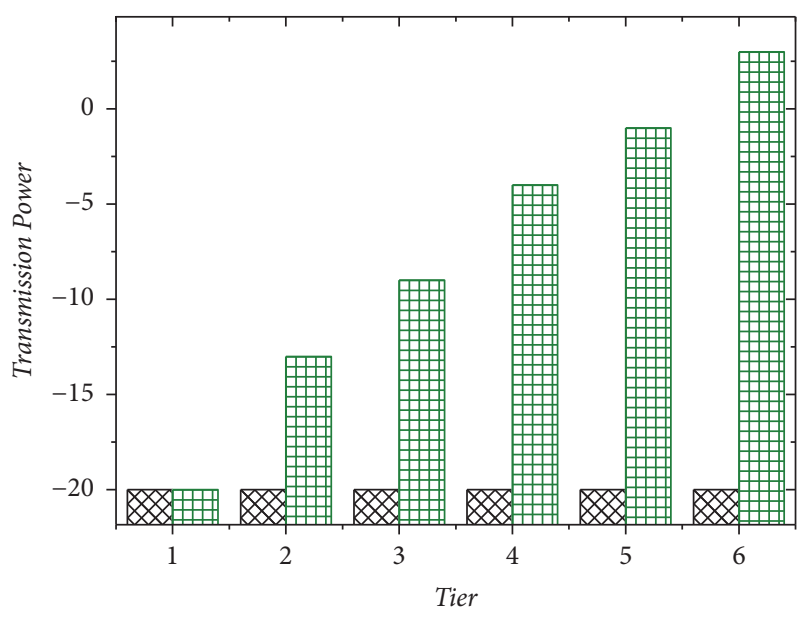

CTPR Scheme 巴田 ATRTC Scheme

FIgURE 12: Comparison of transmission power.

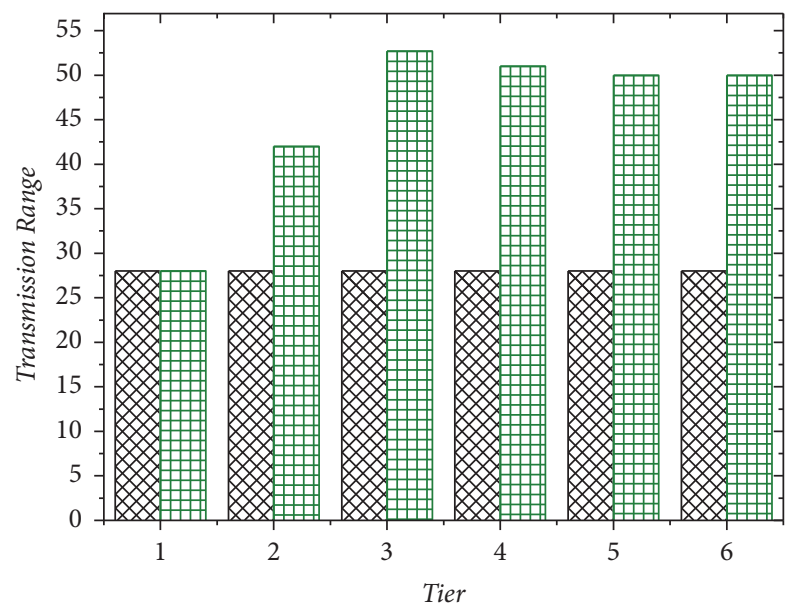

CTPR Scheme

其田 ATRTC Scheme

FIGURE 13: Comparison of transmission range.

improve the transmission reliability of the node. The transmission range of the nodes under CTPR Scheme and ATRTC Scheme is compared in Figure 13. It can be seen from Figure 13 that the transmission range of nodes in all tiers is the same under the CTPR scheme. The transmission range of node is all increased in tier 2-6 under ATRTC scheme. The transmission range of node in tier 2 is $42 \mathrm{~m}$; it can just reach the sink node. The transmission range of node from tier 3 to tier 6 is slightly reduced, because the total area of tier is increased from tier 3 to tier 6 .

Figure 14 shows which tier the child node can reach. The number on the vertical axis represents the serial number of tier, and 0 represents the serial number of sink node tier. Under the CTPR scheme, the serial number of the parent tier is always one less than the serial number of the child tier. The transmission range under CTPR scheme is all the same. But under the ATRTC scheme, most serial number of parent tier 


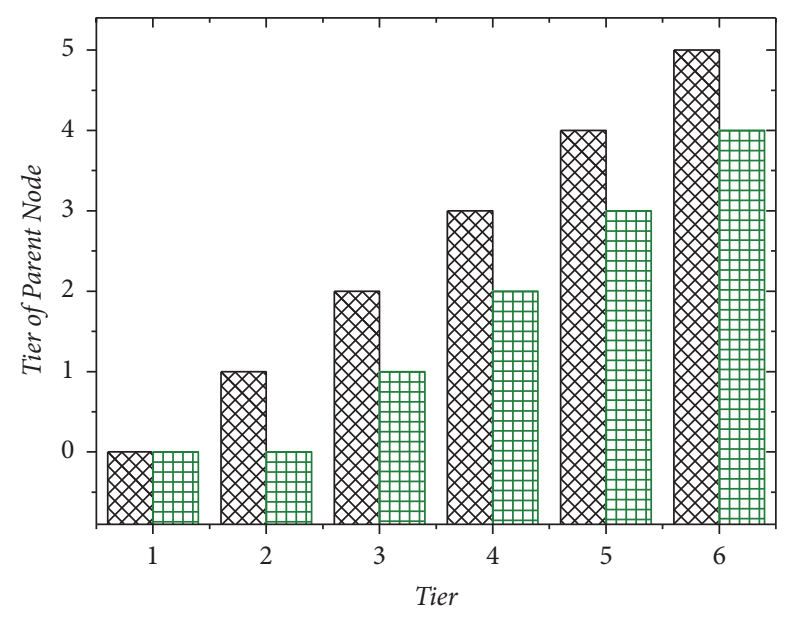

CTPR Scheme \# ATRTC Scheme

FIGURE 14: Comparison of tier of parent node.

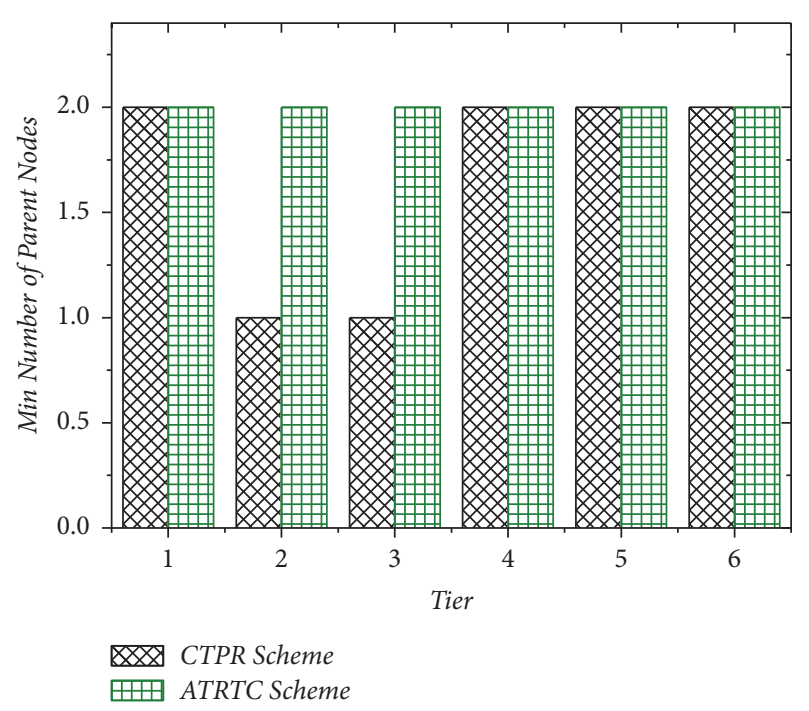

FIGURE 15: Comparison of min number of parent nodes.

is two less than the serial number of the child tier, and nodes in tier 1 and tier 2 have the same serial number of parent tier. Nodes in tier 2 have residual energy and the transmission range of them is extended.

Figure 15 shows the minimum number of parent nodes in each tier under ATRTC scheme and CTPR scheme. Under CTPR scheme, the minimum number of in tier 2 and tier 3 is 1 . If a child node only has only one parent node, broadcast mechanism will not improve the reliability of data transmission from a child node to parent node. Therefore, nodes in tier 2 and tier 3 transmit data using retransmission mechanism to improve the reliability under the CTPR scheme. And under ATRTC scheme, the minimum number of parent nodes in each tier is always 2 due to the increase of transmission range.

Figure 16 shows the path of data collection under ATRTC scheme and CTPR scheme. Under CTPR scheme, a path of data is collected from outer to inner tier by tier. And under the ATRTC scheme, there are two paths of data collection. Nodes in tier 6 transmit data to parent nodes in tier 4 and nodes in tier 5 transmit data to parent nodes in tier 3 . Nodes in tier 6 and tier 5 can transmit data simultaneously. As can be seen in the Figure 16, nodes in tier 6 can transmit data to the sink node just through 3 hops under ATRTC and 5 hops under CTPR scheme.

The average number of parent and average number of children under the CTPR Scheme and ATRTC Scheme are compared in Figures 17 and 18, respectively. It can be seen from Figure 17 that the average number of parent nodes of nodes in tier 4-6 under the ATRTC scheme is increased compared to the CTPR scheme due to the increase of the transmission range. As can be seen from Figure 18, under the ATRTC scheme, the average number of child node in tier 1-4 is more than that under the CTPR scheme. The total number of nodes in a tier is increased with the serial number of tier increase according to Figure 19. And the most serial number of child tier under ATRTC scheme is more than that under CTPR scheme with the same parent tier serial number.

5.2. Data Collection Reliability. In Figure 20, the PRR of node in every tier is 0.868 due to the same transmission range and power under CTPR scheme. And under ATRTC scheme, there is a significantly improvement of the PRR in tiers 4, 5, and 6 compared with CTPR scheme. Because the nodes in tiers 4,5 , and 6 have lots of residual energy, they can use a high transmission power under ATRTC. This led to a high PRR. The PRR of nodes in tier 2 and 3 under ATRTC scheme is roughly equal to that under CTPR scheme. The residual energy of nodes in tiers 2 and 3 is not as much as that in tiers 4,5 , and 6 . The residual energy is used to extend the transmission range at first. The one-hop reliability of nodes under ATRTC scheme and CTPR scheme is compared in Figure 21. Under the ATRTC scheme, the one-hop reliability of nodes in tiers 4,5 , and 6 has a significant improvement compared with CTPR scheme. And the one-hop reliability of nodes in tiers 1,2, and 3 under ATRTC scheme is roughly equal to the CTPR scheme. But the one-hop reliability of nodes can reach 0.98 at least both under ATRTC and CTPR scheme.

The reliability of data reaching sink node (RDS) from nodes in each tier under ATRTC scheme and CTPR scheme is compared in Figure 22. Under the CTPR scheme, the RRS is decreased with the increase of tier serial number. The reliability of tier 6 is the lowest, that is, 0.9002 . This is because every hop of data transmission has a possibility of data loss, and the further away from the sink node, the more hops required. Under the ATRTC scheme, the RDS from nodes in all tiers except tier 1 is higher than that under CTPR scheme. Transmitting data to the sink node under ATRTC scheme needs less hops than CTPR scheme. And the onehop reliability of nodes in most tiers under ATRTC scheme is higher than CTPR scheme. The RDS from node in tier 5 is the lowest under ATRTC scheme, and it still reaches 0.9659 . According to Theorem 13, the reliability of data collection under ATRTC scheme is 0.9659 and under CTPR scheme is 0.9002. The ATRTC scheme increased by $7.3 \%$ over the CTPR scheme on the reliability of data collection. 


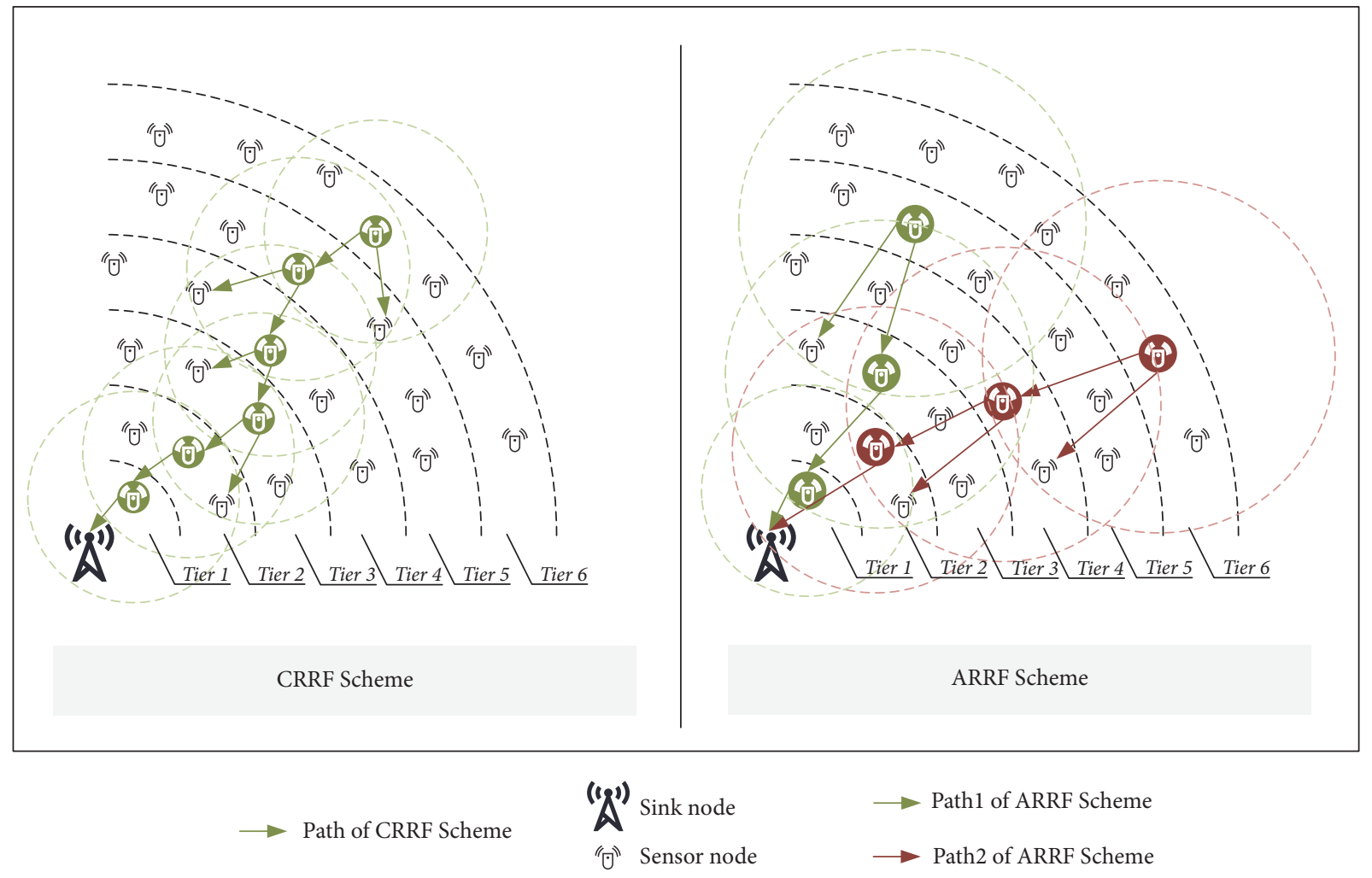

FIGURE 16: Comparison of data collection path.

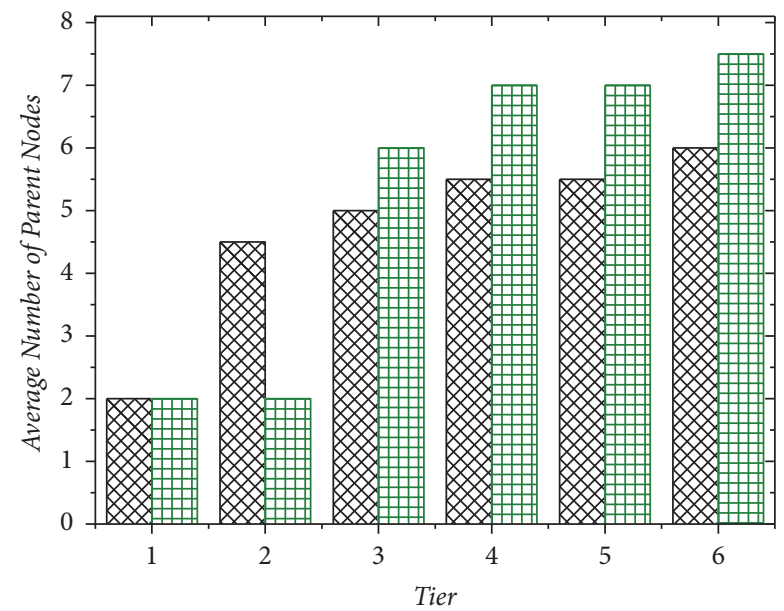

CTPR Scheme 巴田 ATRTC Scheme

FIGURE 17: Comparison of average number of parent nodes.

5.3. Data Collection Time. The sending data volume and receiving data volume of nodes in each tier under ATRTC scheme and CTPR scheme are shown in Figures 23 and 24, respectively. In Figure 23, the sending data volume of nodes increases as the serial number of tiers decreases. The parent nodes in the inner tier need to forward the data of the child nodes in outer tier and the number of parent nodes is less than the number of child nodes. But under ATRTC scheme the sending data volume is less than CTPR scheme.

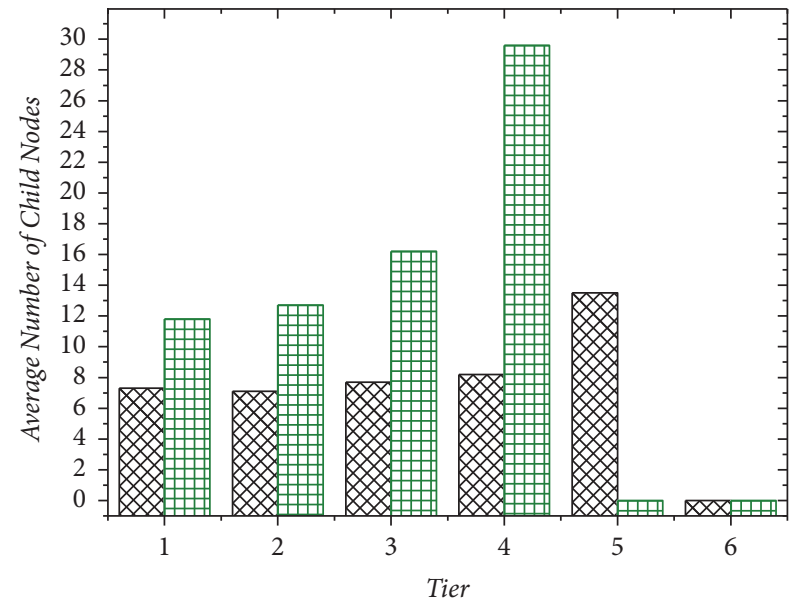

CTPR Scheme 田田 ATRTC Scheme

FIGURE 18: Comparison of average number of child nodes.

As can be seen from Figure 24, under the ATRTC scheme, the receiving data volume of nodes in most tiers is less than the CTPR scheme. But the receiving data volume of nodes in tier 2 is more than the CTPR scheme. And the number of child nodes under ATRTC scheme is more than CTPR scheme and the sending data volume of child nodes is roughly the same according to Figures 18 and 23.

Tables 4 and 5 show the paths of one-hop and the time spent on them under CTPR scheme and ATRTC scheme. As 


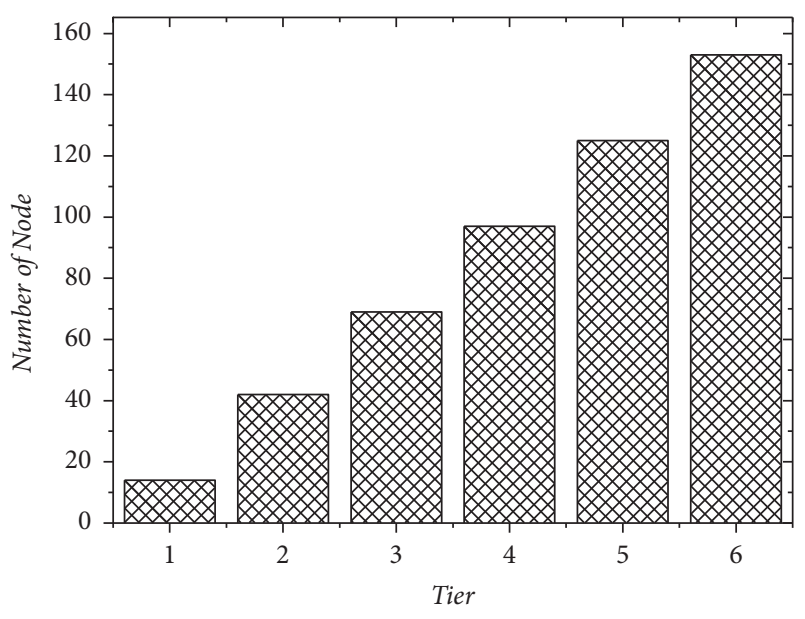

FIGURE 19: Number of node in each tier.

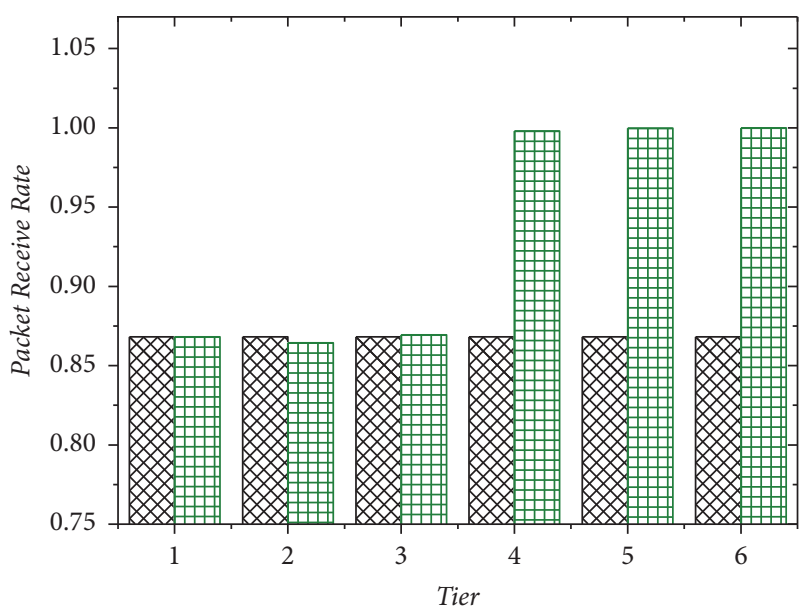

CTPR Scheme

\#田 ATRTC Scheme

FIGURE 20: Comparison of packet receive rate.

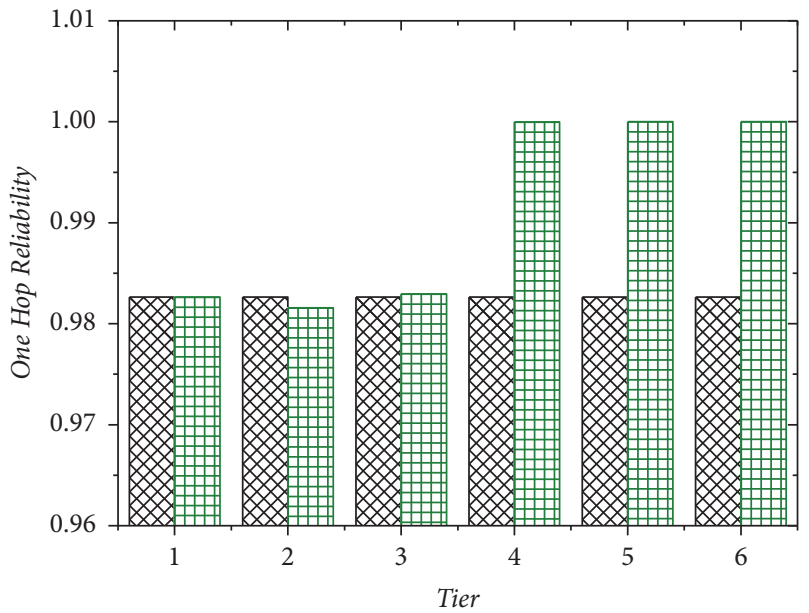

CTPR Scheme 巴 ATRTC Scheme

FIGURE 21: Comparison of one-hop reliability.

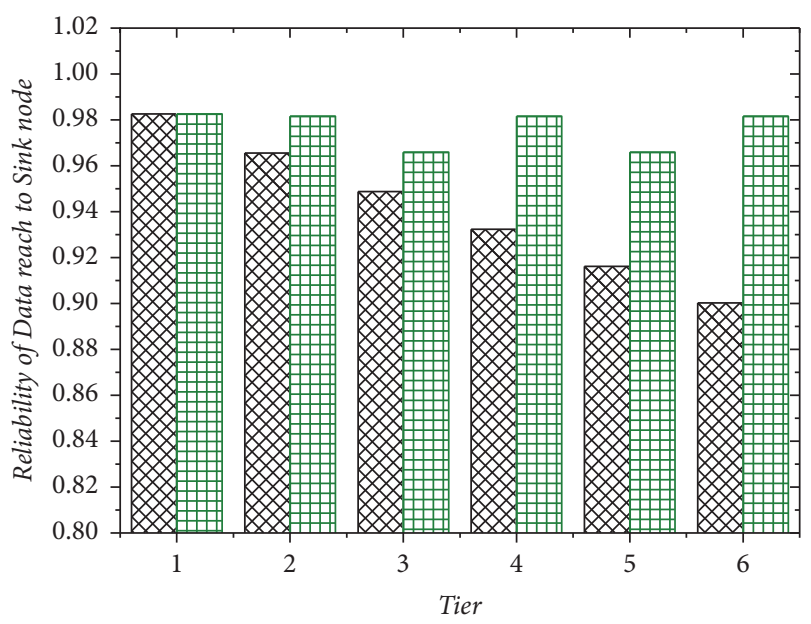

CTPR Scheme 巴 ATRTC Scheme

FIGURE 22: Comparison of reliability of data transmit to sink.

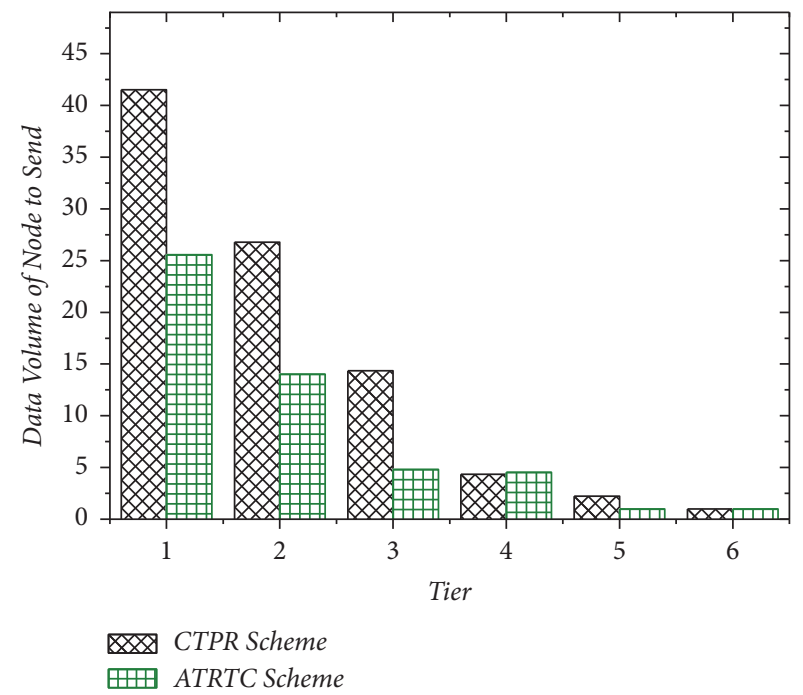

FIgURE 23: Comparison of data volume of node to send.

TABLE 4: Data collection time in CTPR scheme.

\begin{tabular}{lcc}
\hline Serial number & Path of one hop & Time $(s)$ \\
\hline $\mathbf{1}$ & $6->5$ & 0.152083 \\
$\mathbf{2}$ & $5->4$ & 0.329560 \\
$\mathbf{3}$ & $4->3$ & 0.695604 \\
$\mathbf{4}$ & $3->2$ & 1.226709 \\
$\mathbf{5}$ & $2->1$ & 3.766472 \\
$\mathbf{6}$ & $1->0$ & 12.112338 \\
\hline \multicolumn{3}{c}{ Data collection time } \\
\hline
\end{tabular}

can be seen from Figures 4 and 5 , the closer to the sink node, the more time which is spent on the path of one-hop. The closer to the sink node, the more time which is spent on the one-hop path. Under the same serial number, the time spent on the path of one-hop under ATRTC scheme is mostly more 


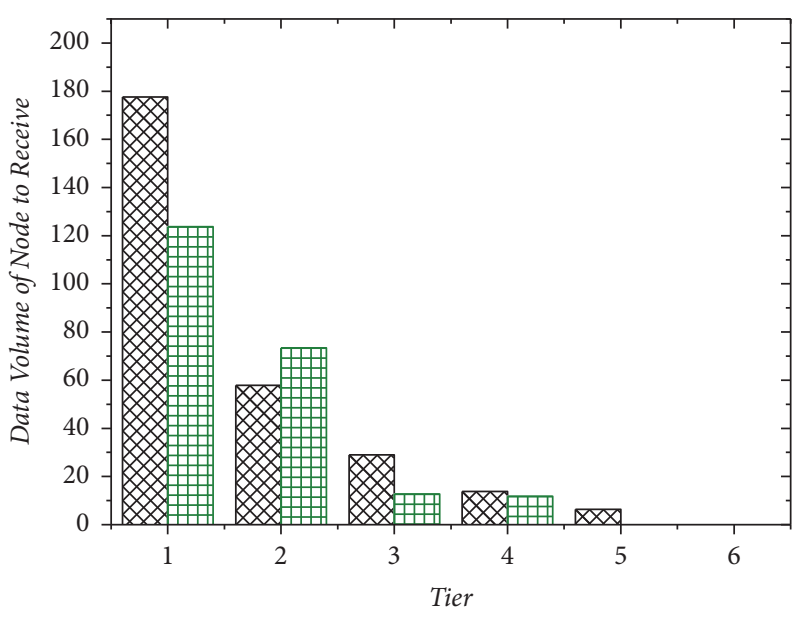

CTPR Scheme 曲 ATRTC Scheme

Figure 24: Comparison of data volume of node to receive.

TABLE 5: Data collection time in ATRTC scheme.

\begin{tabular}{lcc}
\hline Serial number & Path of one hop & Time(s) \\
\hline $\mathbf{1}$ & $6->4$ & 0.245833 \\
$\mathbf{2}$ & $5->3$ & 0.264583 \\
$\mathbf{3}$ & $4->2$ & 1.532247 \\
$\mathbf{4}$ & $3->1$ & 2.966007 \\
$\mathbf{5}$ & $2->0$ & 12.278143 \\
$\mathbf{6}$ & $1->0$ & 7.459946 \\
\hline \multicolumn{3}{c}{ Data collection time } \\
\hline
\end{tabular}

than CTPR scheme. For example, when serial number is 1 , under CTPR scheme the time spent on one-hop is $0.152083 \mathrm{~s}$ and 0.245833 s under ATRTC scheme. But under ATRTC scheme, data from the nodes in tier 6 can directly reach the nodes in tier 4 in 0.245833 s and 0.481643 s under CTPR scheme. This is because of the cross-tier transmission and two paths of data collection under ATRTC scheme. Therefore, the data collection time of the network under the ATRTC scheme was reduced by $23 \%$ compared to the CTPR scheme.

5.4. Energy Consumption and Network Lifetime. The energy consumption of nodes in each tier and network lifetime under CTPR scheme and ATRTC scheme is compared in this section. The energy consumption for sending data, the energy consumption for receiving data, and the total energy consumption of nodes in each tier in a data collection cycle are shown in Figures 25, 26, and 27, respectively. As shown in Figure 25, the energy consumption of nodes in tier 1 under ATRTC scheme is lower than CTPR scheme, and the energy consumption of nodes in tiers 2-6 under ATRTC scheme is higher than CTPR scheme. Because the nodes in tiers 2-6 have residual energy, the transmission power of them is increased under ATRTC scheme. The trend of Figure 26 is similar to Figure 24, because the receiving power is not changed under ATRTC scheme. As shown in Figure 27, the closer to the sink node, the greater the energy consumption

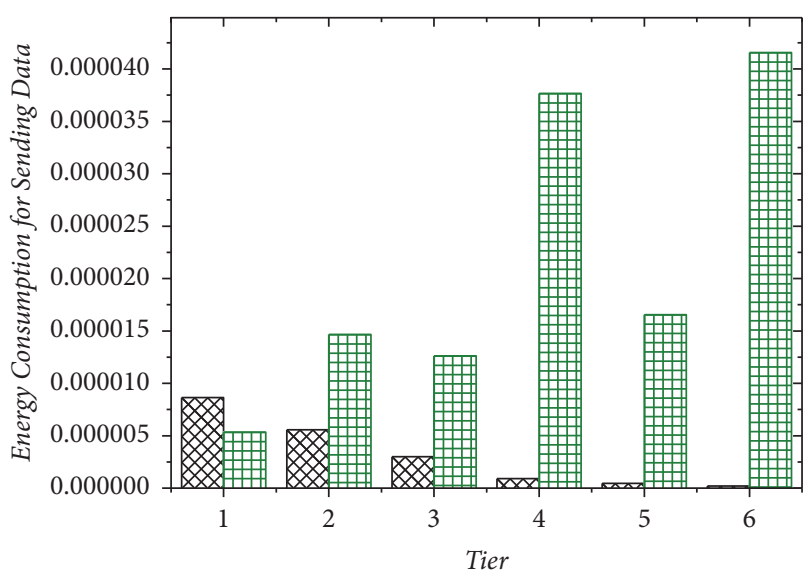

CTPR Scheme 囘 ATRTC Scheme

FIGURE 25: Comparison of energy consumption for sending data.

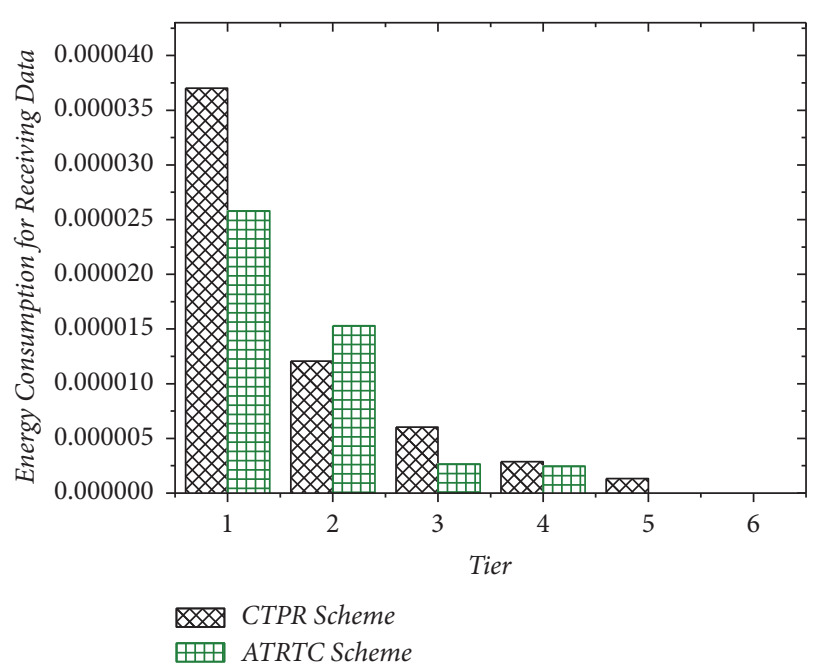

FIGURE 26: Comparison of energy consumption for receiving data.

TABLE 6: Lifetime under ATRTC scheme and CTPR scheme.

\begin{tabular}{lcc}
\hline Tier & $\begin{array}{c}\text { Lifetime under } \\
\text { CTPR Scheme }\end{array}$ & $\begin{array}{c}\text { Lifetime under } \\
\text { ATRTC Scheme }\end{array}$ \\
\hline $\mathbf{1}$ & 43799 & 64274 \\
$\mathbf{2}$ & 113417 & 66790 \\
$\mathbf{3}$ & 221450 & 131059 \\
$\mathbf{4}$ & 531251 & 49859 \\
$\mathbf{5}$ & 1120706 & 120856 \\
$\mathbf{6}$ & 9600000 & 48113 \\
\hline Network Lifetime & 43799 & 48113 \\
\hline
\end{tabular}

of nodes under the CTPR scheme. The maximum and minimum ratio of energy consumption is 219.2 under CTPR scheme and 2.7 under ATRTC scheme. And the maximum energy consumption under ATRTC scheme is 9\% less than CTPR scheme. 


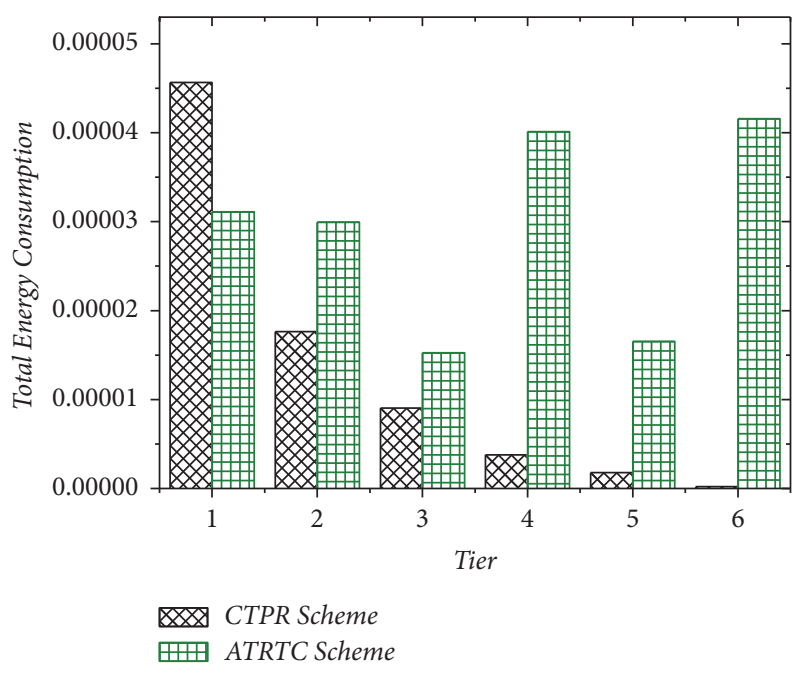

FIGURE 27: Comparison of total energy consumption.

Table 6 shows the lifetime of nodes in each tier under ATRTC scheme and CTPR scheme. According to the definition of the network lifetime, it is the minimum lifetime of node. The network lifetime under CTPR scheme is 43799 cycles and 48113 cycles under ATRTC scheme. The network lifetime under ATRTC scheme is about $10 \%$ higher than CTPR scheme.

\section{Conclusion}

In the wireless sensor network, the resources of a node are quite limited, and the wireless channel is lossy. Therefore, how to achieve high stability, low power consumption, and low latency network performance is very challenging under limited energy conditions.

This paper focuses on optimizing the lifetime, data collection time, and data collection reliability of periodic data collection wireless sensor networks by controlling the transmission range of the nodes. After careful analysis, we found that the traditional Constant Transmission Power and Range (CTPR) scheme has a shortage that energy consumption near-sink node is much higher than far-sink node. Therefore, the Adaptive Transmission Range Based Topology Control (ATRTC) scheme is proposed in this paper. If a node has residual energy, the transmission power will be increased, the transmission range will be extended, and the transmission quality will be improved under ATRTC scheme. Increasing the transmission range can reduce the number of hops that data reaches the sink node and improve the reliability of data collection. And the increase of paths of data collection leads to reducing of data collection time. According to the analysis in this paper, compared with the CTPR scheme, the ATRTC scheme reduces the maximum energy consumption by $9 \%$, increases the network lifetime by $10 \%$, increases the data collection reliability by $7.3 \%$, and reduces the network data collection time by $23 \%$.

The ATRTC scheme also has some limitations. If the energy consumption difference between the nodes is not very large, the nodes do not have enough residual energy to substantially extend the transmission range to achieve crosstier transmission. In this case, the ATRTC scheme will not have a significant performance increase. How to improve the performance in that case can be solved in future work. In addition, ATRTC scheme improves the transmission range of sensor nodes. And larger transmission range will be interfered by more nodes. How to reduce the interference between nodes is also a problem that needs to be solved in the future.

\section{Data Availability}

This paper does not include data which can be shared with the public. The data used to support the findings of this study have been described in this paper clearly.

\section{Conflicts of Interest}

The authors declare that there are no conflicts of interest regarding the publication of this article.

\section{Acknowledgments}

This work was supported in part by the National Natural Science Foundation of China (61772554, 61572526, and 61572528), the Open Research Project of the State Key Laboratory of Industrial Control Technology, Zhejiang University, China (no. ICT1800391), the National Basic Research Program of China (973 Program) (2014CB046305), JSPS KAKENHI Grant nos. JP16K00117 and JP15K15976, and KDDI Foundation.

\section{References}

[1] A. Liu, M. Huang, M. Zhao, and T. Wang, "A Smart High-Speed Backbone Path Construction Approach for Energy and Delay Optimization in WSNs," IEEE Access, vol. 6, pp. 13836-13854, 2018.

[2] M. Huang, A. Liu, M. Zhao, and T. Wang, "Multi working sets alternate covering scheme for continuous partial coverage in WSNs," Peer-to-Peer Networking and Applications, 2018.

[3] P. L. Nguyen, Y. Ji, Z. Liu, H. Vu, and K.-V. Nguyen, "Distributed hole-bypassing protocol in WSNs with constant stretch and load balancing," Computer Networks, vol. 129, pp. 232-250, 2017.

[4] Z. Liu, T. Tsuda, H. Watanabe, S. Ryuo, and N. Iwasawa, "Data Driven Cyber-Physical System for Landslide Detection," Mobile Networks and Applications, 2018.

[5] K. Ota, M. S. Dao, V. Mezaris, and F. G. Natale, "Deep Learning for Mobile Multimedia," ACM Transactions on Multimedia Computing, Communications, and Applications (TOMM), vol. 13, no. 3s, pp. 1-22, 2017.

[6] Y. Zhang, X. Chen, J. Li, D. S. Wong, H. Li, and I. You, "Ensuring attribute privacy protection and fast decryption for outsourced data security in mobile cloud computing," Information Sciences, vol. 379, pp. 42-61, 2017.

[7] Q. Xu, Z. Su, and K. Yang, "Optimal control theory-based epidemic information spreading scheme for mobile social users with energy constraint," IEEE Access, vol. 5, pp. 14107-14117, 2017.

[8] Q. Xu, Z. Su, Q. Zheng, M. Luo, and B. Dong, "Secure Content Delivery with Edge Nodes to Save Caching Resources for Mobile Users in Green Cities," IEEE Transactions on Industrial Informatics, 2017. 
[9] M. Li, Z. Li, and A. V. Vasilakos, "A survey on topology control in wireless sensor networks: taxonomy, comparative study, and open issues," Proceedings of the IEEE, vol. 101, no. 12, pp. 25382557, 2013.

[10] X. Liu, "A novel transmission range adjustment strategy for energy hole avoiding in wireless sensor networks," Journal of Network and Computer Applications, vol. 67, pp. 43-52, 2016.

[11] Y. Liu, A. Liu, S. Guo, Z. Li, Y.-J. Choi, and H. Sekiya, "Contextaware collect data with energy efficient in Cyber-physical cloud systems," Future Generation Computer Systems, 2017.

[12] K. Xie, J. Cao, X. Wang, and J. Wen, "Optimal resource allocation for reliable and energy efficient cooperative communications," IEEE Transactions on Wireless Communications, vol. 12, no. 10, pp. 4994-5007, 2013.

[13] Y. Liu, K. Ota, K. Zhang et al., "QTSAC: An Energy-Efficient MAC Protocol for Delay Minimization in Wireless Sensor Networks," IEEE Access, vol. 6, pp. 8273-8291, 2018.

[14] H. Dai, G. Chen, C. Wang, S. Wang, X. Wu, and F. Wu, "Quality of energy provisioning for wireless power transfer," IEEE Transactions on Parallel and Distributed Systems, vol. 26, no. 2, pp. 527-537, 2015.

[15] J. Tang, A. Liu, J. Zhang, N. Xiong, Z. Zeng, and T. Wang, "A Trust-Based Secure Routing Scheme Using the Traceback Approach for Energy-Harvesting Wireless Sensor Networks," Sensors, vol. 18, no. 3, p. 751, 2018.

[16] T. Han and N. Ansari, "Network Utility Aware Traffic Load Balancing in Backhaul-Constrained Cache-Enabled Small Cell Networks with Hybrid Power Supplies," IEEE Transactions on Mobile Computing, vol. 16, no. 10, pp. 2819-2832, 2017.

[17] Y. Guo, F. Liu, Z. Cai, N. Xiao, and Z. Zhao, "Edge-Based Efficient Search over Encrypted Data Mobile Cloud Storage," Sensors, vol. 18, no. 4, p. 1189, 2018.

[18] X. Liu, G. Li, S. Zhang, and A. Liu, "Big program code dissemination scheme for emergency software-define wireless sensor networks," Peer-to-Peer Networking and Applications, vol. 11, no. 5, pp. 1038-1059, 2018.

[19] S. Narayanaswamy, V. Kawadia, R. S. Sreenivas, and P. R. Kumar, "Power Control in Ad-Hoc Networks: Theory, Architecture," in Proceedings of the Power Control in Ad-Hoc Networks: Theory, Architecture, pp. 156-162, 2002.

[20] H. Xin and X. Liu, "Energy-balanced transmission with accurate distances for strip-based wireless sensor networks," IEEE Access, vol. 5, pp. 16193-16204, 2017.

[21] J. Xu, A. Liu, N. Xiong, T. Wang, and Z. Zuo, "Integrated collaborative filtering recommendation in social cyber-physical systems," International Journal of Distributed Sensor Networks, vol. 13, no. 12, p. 155014771774974, 2017.

[22] J. Cui, Y. Zhang, Z. Cai, A. Liu, and Y. Li, "Securing Display Path for Security-Sensitive Applications on Mobile Devices," CMC, Computers, Materials \& Continua, vol. 55, no. 1, pp. 17-035, 2018.

[23] A. Liu, W. Chen, and X. Liu, "Delay optimal opportunistic pipeline routing scheme for cognitive radio sensor networks," International Journal of Distributed Sensor Networks, vol. 14, no. 4, p. $155014771877253,2018$.

[24] Z. Li, B. Chang, S. Wang, A. Liu, F. Zeng, and G. Luo, "Dynamic Compressive Wide-Band Spectrum Sensing Based on Channel Energy Reconstruction in Cognitive Internet of Things," IEEE Transactions on Industrial Informatics, vol. 14, no. 6, pp. 25982607, 2018.

[25] M. Z. A. Bhuiyan, G. Wang, J. Wu, J. Cao, X. Liu, and T. Wang, "Dependable Structural Health Monitoring Using Wireless
Sensor Networks," IEEE Transactions on Dependable and Secure Computing, vol. 14, no. 4, pp. 363-376, 2017.

[26] M. Huang, Y. Liu, N. Zhang et al., "A Services Routing Based Caching Scheme for Cloud Assisted CRNs," IEEE Access, vol. 6, pp. 15787-15805, 2018.

[27] X. Liu, S. Zhao, A. Liu, N. Xiong, and A. V. Vasilakos, "Knowledge-aware Proactive Nodes Selection approach for energy management in Internet of Things," Future Generation Computer Systems, 2017.

[28] W. Sun, Z. Cai, Y. Li, F. Liu, S. Fang, and G. Data, "Data Proessing and Text Mining Technologies on Electronic Medical Records: A Review," Journal of Healthcare Engineering, Article ID 4302425, 2018.

[29] Y. Liu, M. Dong, K. Ota, and A. Liu, "ActiveTrust: secure and trustable routing in wireless sensor networks," IEEE Transactions on Information Forensics and Security, vol. 11, no. 9, pp. 2013-2027, 2016.

[30] X. Liu, M. Dong, Y. Liu, A. Liu, and N. Xiong, "Construction Low Complexity and Low Delay CDS for Big Data Codes Dissemination," Complexity, vol. 2018, Article ID 5429546, 10 pages, 2018.

[31] X. Liu, N. Xiong, N. Zhang, A. Liu, H. Shen, and C. Huang, "A trust with abstract information verified routing scheme for cyber-physical network," IEEE Access, pp. 3882-3898, 2018.

[32] M. Z. A. Bhuiyan, J. Wu, G. Wang, T. Wang, and M. M. Hassan, "e-Sampling: Event-sensitive autonomous adaptive sensing and low-cost monitoring in networked sensing systems," ACM Transactions on Autonomous and Adaptive Systems (TAAS), vol. 12, no. 1, article no. 1, 2017.

[33] S. Zhao and A. Liu, "High performance target tracking scheme with low prediction precision requirement in WSNs," International Journal of Ad Hoc and Ubiquitous Computing, 2017, http://www.inderscience.com/info/ingeneral/forthcoming.php? jcode=ijahuc.

[34] T. Wang, J. Zhou, M. Huang et al., "Fog-based storage technology to fight with cyber threat," Future Generation Computer Systems, vol. 83, pp. 208-218, 2018.

[35] J. Li, X. Chen, F. Xhafa, and L. Barolli, "Secure deduplication storage systems supporting keyword search," Journal of Computer and System Sciences, vol. 81, no. 8, pp. 1532-1541, 2015.

[36] W. Jiang, G. Wang, M. Z. Bhuiyan, and J. Wu, "Understanding Graph-Based Trust Evaluation in Online Social Networks," ACM Computing Surveys, vol. 49, no. 1, pp. 1-35, 2016.

[37] X. Chen, J. Li, J. Weng, J. Ma, and W. Lou, "Verifiable computation over large database with incremental updates," Institute of Electrical and Electronics Engineers. Transactions on Computers, vol. 65, no. 10, pp. 3184-3195, 2016.

[38] J. Li, Z. Liu, X. Chen, F. Xhafa, X. Tan, and D. S. Wong, "LEncDB: A lightweight framework for privacy-preserving data queries in cloud computing," Knowledge-Based Systems, vol. 79, pp. 18-26, 2015.

[39] X. Liu, Y. Liu, N. N. Xiong et al., "Construction of Large-Scale Low-Cost Delivery Infrastructure Using Vehicular Networks," IEEE Access, vol. 6, pp. 21482-21497, 2018.

[40] S. Lin, J. Zhang, G. Zhou, L. Gu, J. A. Stankovic, and T. He, "ATPC: adaptive transmission power control for wireless sensor networks," in Proceedings of the Proceeding of the 4th International Conference on Embedded Networked Sensor Systems (SenSys '06), pp. 223-236, New York, NY, USA, November 2006.

[41] M. Zuniga and B. Krishnamachari, "An analysis of unreliability and asymmetry in low-power wireless links," in Proceedings of 
the 1st Annual IEEE Communications Society Conference on Sensor and Ad Hoc Communications and Networks (IEEE SECON '04), pp. 517-526, October 2004.

[42] Y.-C. Tseng, S.-Y. Ni, Y.-S. Chen, and J.-P. Sheu, "The broadcast storm problem in a mobile ad hoc network," Wireless Networks, vol. 8, no. 2-3, pp. 153-167, 2002.

[43] M. T. Sun and T.-H. Lai, "Location aided broadcast in wireless ad hoc network systems," in Proceeding of the IEEE Wireless Communications and Networking Conference Record (WCNC '02), vol. 2, pp. 597-602, 2002.

[44] J. Wu and F. Dai, "A generic distributed broadcast scheme in ad hoc wireless networks," IEEE Transactions on Computers, vol. 53, no. 10, pp. 1343-1354, 2004.

[45] C. Joo and N. B. Shroff, "On the delay performance of in-network aggregation in lossy wireless sensor networks," IEEE/ACM Transactions on Networking, vol. 22, no. 2, pp. 662-673, 2014.

[46] P. Jesus, C. Baquero, and P. S. Almeida, "A Survey of Distributed Data Aggregation Algorithms," IEEE Communications Surveys \& Tutorials, vol. 17, no. 1, pp. 381-404, 2015.

[47] X. Li, A. Liu, M. Xie, N. Xiong, Z. Zeng, and Z. Cai, "Adaptive Aggregation Routing to Reduce Delay for Multi-Layer Wireless Sensor Networks," Sensors, vol. 18, no. 4, p. 1216, 2018.

[48] Z. Iqbal and H. Lee, "Low-Latency and High-Reliability Cooperative WSN for Indoor Industrial Monitoring," in Proceedings of the 2017 IEEE 85th Vehicular Technology Conference (VTC Spring), pp. 1-6, Sydney, NSW, June 2017.

[49] B. Kang, P. K. H. Nguyen, V. Zalyubovskiy, and H. Choo, "A Distributed Delay-Efficient Data Aggregation Scheduling for Duty-Cycled WSNs,' IEEE Sensors Journal, vol. 17, no. 11, pp. 3422-3437, 2017.

[50] C.-Y. Wan, A. T. Campbell, and L. Krishnamurthy, "PSFQ: A reliable transport protocol for wireless sensor networks," in Proceedings of the 1st ACM International Workshop on Wireless Sensor Networks and Applications, pp. 1-11, USA, September 2002.

[51] S. Park, R. Vedantham, R. Sivakumar, and I. F. Akyildiz, "A scalable approach for reliable downstream data delivery in wireless sensor networks," in Proceedings of the the 5th ACM international symposium, p. 78, Roppongi Hills, Tokyo, Japan, May 2004.

[52] F. Xiao, W. Liu, Z. Li, L. Chen, and R. Wang, "Noise-tolerant wireless sensor networks localization via multi-norms regularized matrix completion," IEEE Transactions on Vehicular Technology, vol. 67, no. 3, pp. 2409-2419, 2018.

[53] H. Wen, C. Lin, F. Ren, Y. Yue, and X. Huang, "Retransmission or redundancy: Transmission reliability in wireless sensor networks," in Proceedings of the IEEE Internatonal Conference on Mobile Adhoc and Sensor Systems, (MASS' 07), Italy, October 2007.

[54] H. Teng, X. Liu, A. Liu, H. Shen, C. Huang, and T. Wang, "Adaptive Transmission Power Control for Reliable Data Forwarding in Sensor Based Networks," Wireless Communications and Mobile Computing, vol. 2018, 2018. 


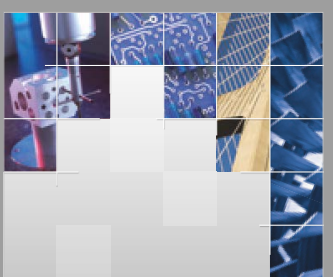

\section{Enfincering}
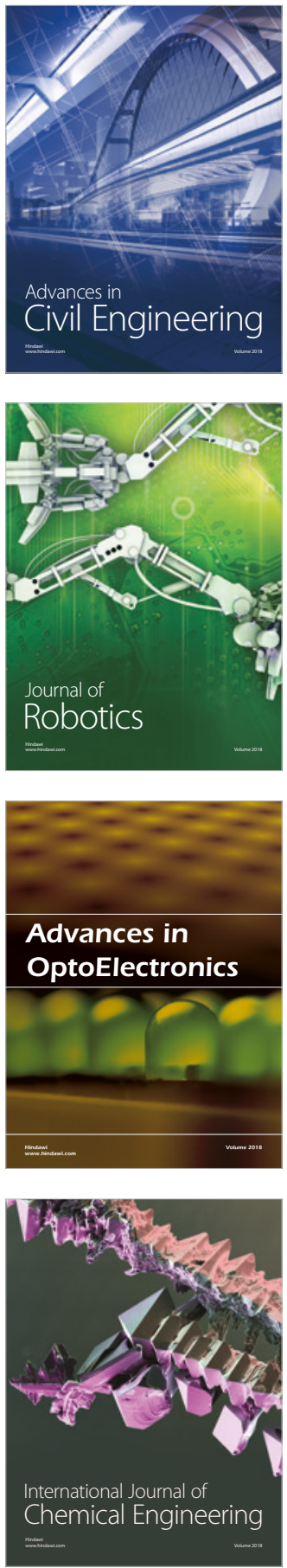

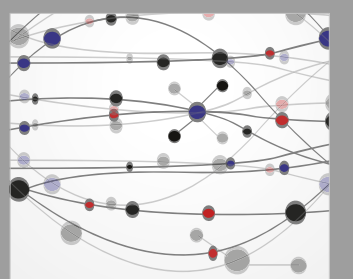

\section{Rotating \\ Machinery}

The Scientific World Journal

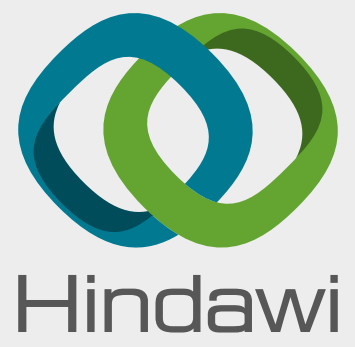

Submit your manuscripts at

www.hindawi.com
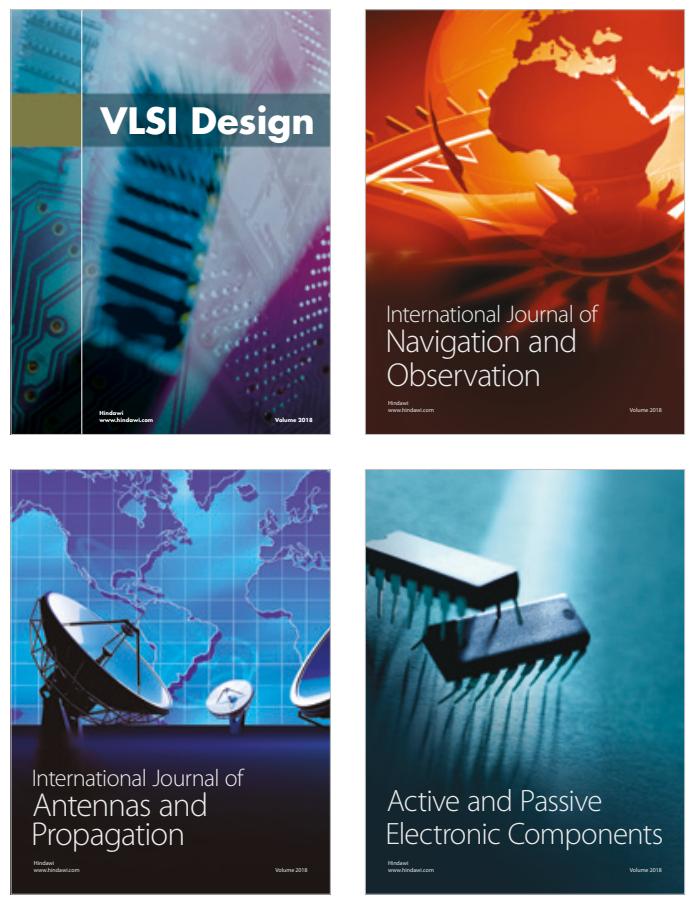
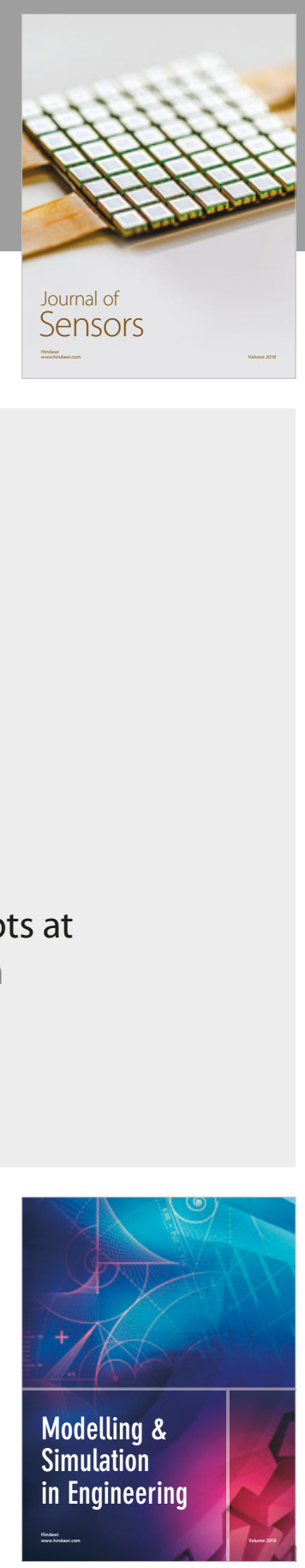

\section{Advances \\ Multimedia}
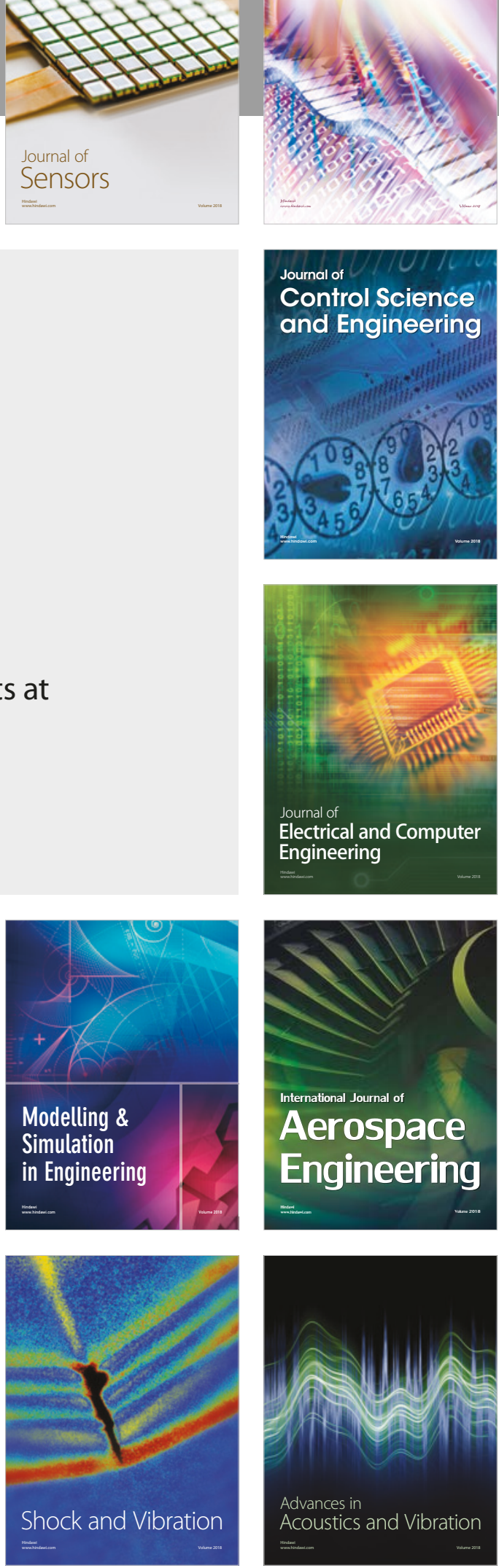